\title{
Multicast Wi-Fi Raptor-enabled data carousel design: simulation and practical implementation
}

\author{
Berna Bulut ${ }^{1 *}$, Denys Berkovskyy ${ }^{1}$, Siming Zhang ${ }^{1}$, Michael Collett ${ }^{1}$, Evangelos Mellios ${ }^{1}$, David Armour ${ }^{2}$,
} Angela Doufexi ${ }^{1}$ and Andrew Nix ${ }^{1}$

\begin{abstract}
Multicast is an efficient way of transmitting the same set of data to multiple interested users. Unlike the 3rd Generation Partnership Project (3GPP) cellular standards, for Wi-Fi, there is no standardised solution for reliable multicast data transmission. Multicast packets are delivered to multiple users as a broadcast service without support for automatic repeat request. Hence, multicast transmission often results in high packet loss. In order to improve the reliability of multicast delivery, a fixed low-speed (robust) transmission mode can be used. However, this results in the inefficient use of scarce and valuable radio bandwidth. This paper presents a reliable and efficient Wi-Fi multicast delivery solution for use in challenging outdoor environments. An application layer forward error correction (AL-FEC)-enabled data carousel is proposed to enhance reliability. For multicast transmission, we demonstrate that limitations in the Wi-Fi clients are a major source of packet loss, even in ideal channel conditions. Client limitations (particularly data rate limitations) were found to vary as a function of modulation and coding mode, Raptor code parameters and multicast server rate. Our initial Raptor-enabled carousel designs are based on computer simulations and lab-based trials. Analysis is then extended to field trials using a practical implementation of the recommended design. These trials were performed in central Bristol with parameters such as received signal level, packet loss traces and file download times recorded at the clients. Finally, we compare our site-specific simulated results against real-world measurements.
\end{abstract}

Keywords: Data carousel, Forward error correction, IEEE 802.11, Multicast, Raptor, RaptorQ, Wi-Fi measurements

\section{Introduction}

The wide availability of cell phones and tablet computers has led to an increase in the demand for mobile multimedia applications. Unicast protocols struggle to meet these demands since the radio and network resources are shared between the users. For unicast transmissions, each user is sent a unique copy of the media. As a consequence, for dense user groups, the network rapidly runs out of bandwidth. The problem is made worse since each unicast user also requests the retransmission of lost data packets via the return channel. While this provides a reliable link, it prevents the widespread dissemination of media-rich content to large numbers of users. One solution to efficiently disseminate high-

\footnotetext{
*Correspondence: berna.bulut@bristol.ac.uk

${ }^{1}$ Department of Electrical and Electronic Engineering, Communications Systems and Networks Group, University of Bristol, Bristol BS8 1UB, UK Full list of author information is available at the end of the article
}

bandwidth media-rich content to many users over errorprone wireless channels is to use multicast transmission. However, standard multicast 802.11 transmissions fail to provide users with a reliable data delivery service.

At present, IEEE 802.11 [1] offers no standardised or certified extension for reliable multicast delivery. Multicast packets are sent as a simple broadcast service without support for automatic repeat request (ARQ). When combined with mobile handsets and tablet computers, where further bottlenecks and restrictions exist, multicast transmission can result in high packet loss rates. Another issue with multicast transmission over IEEE 802.11 networks is that adaptive modulation and coding is unsupported. In practice, to improve reliability, multicast transmission often uses the lowest IEEE 802.11 link speed regardless of channel conditions. This approach is very wasteful of valuable radio spectrum [2].

\section{Springer}


In scenarios where a return channel is unavailable, it is well known that a data carousel or broadcast disk [3] approach can be used to provide reliable multicast file delivery over an error-prone network. With a data carousel, the transmitter continually transmits all the data packets in a cyclic fashion. Receivers may join the carousel at any time and normally leave only when they have received all the packets that belong to the desired file(s). However, wireless communication channels are prone to errors (which result in lost packets) and, as a consequence, users may not obtain all elements of the required file(s) in a single transmission cycle. In such cases, the users must wait for the next carousel cycle to successfully retrieve the file. This approach may result in numerous duplicate packets at each user and a significant increase in the total time required to download the desired media. Application layer forward error correction (AL-FEC) based on traditional block codes can be used in conjunction with data carousels to reduce download time as reported in [4-7]. However, traditional codes suffer from constraints such as a fixed code rate that must be defined beforehand. Furthermore, prior knowledge of the channel conditions is required. If the code rate is underestimated, this approach may still result in the reception of duplicate packets at the users, i.e. limited number of redundant packets.

An ideal solution to the problems listed above is the use of digital fountain (rateless) codes as described in [8]. In terms of providing reliable and scalable multicast, fountain codes are more efficient than any other type of FEC [8]. Unlike traditional block codes, a digital fountain code can generate endless encoded packets from a given source block such that each transmit packet is different and useful for decoding [9]. Rateless codes enable multiple receivers to recover their individual lost packets in a wireless multicast network without the need for individual retransmissions, thus efficiently utilising the radio and network resources. The theory presented here is also applicable to the field of network coding [10], where source data are coded before being forwarded through independent network paths.

Raptor codes [11] are a form of fountain code that operate close to the ideal performance bound. There are two commercially available Raptor codes: Raptor 10 (R10) and RaptorQ (RQ) [12]. R10 has already been integrated into many standards, such as the 3GPP multimedia broadcast and multicast service (MBMS) [13] and the digital video broadcasting-handheld (DVB-H) service [14] (with the exception of wireless local area network (WLAN)) in order to provide robust multicast transmissions. This paper proposes and then designs a Raptor code-enabled data carousel as a reliable multicast data transmission scheme for IEEE 802.11 WLANs in outdoor environments.
We present a number of key contributions. Firstly, we propose an interleaved data carousel model that is combined with the latest RQ (since it offers improved coding efficiency compared to R10) [12]. The interleaved model mitigates against bursts of errors in a source block and hence reduces the time required to acquire all the packets that form the source block. Secondly, we present an analytical model that allows the calculation of file download times for a fountain code-enhanced data carousel. Thirdly, a realistic multi-layered simulator has been developed combining novel outdoor ray tracing, a physical (PHY) layer Wi-Fi simulator and a RQ-enabled multicast data carousel simulator. Limitations in the client (which are not predicted in our theoretic simulations) are shown to result in significant packet loss, even in ideal channel conditions. By implementing our proposed multicast carousel system (server, Wi-Fi access point and client), we show using Android-enabled tablets that real-world packet loss is a strong function of modulation and coding scheme (MCS), Raptor code parameters and multicast server rate. We recommend a full set of design parameters based on a combination of our theoretic simulations and practical tablet-based measurements. Finally, we validate and evaluate the performance of the proposed system and via a measurement campaign conducted on the streets of Bristol, UK.

The rest of the paper is organised as follows. Section 2 provides an overview of Raptor code AL-FEC and data carousels. Section 3 explains the FEC data carousel model. Problem formulation is given in Section 4. Section 5 details the evaluation methodologies for our simulations and hardware measurements. Section 6 presents evaluation parameters. Results and analysis are presented in Section 7. Finally, Section 8 concludes the paper.

\section{Background}

\subsection{Raptor codes}

Raptor codes are a form of fountain code that can generate an unlimited number of encoded symbols on-the-fly from a fixed source block. Due to this property, these codes are characterized as rateless codes. In a fountain code, it does not matter which particular symbols are received, as long as a sufficient number of symbols arrive.

The main drawback of Raptor codes is that the decoder needs slightly more symbols than the original $k$ source symbols to reconstruct the file, implying that Raptor codes have a small reception overhead. This is defined as $\varepsilon=r-k$, where $r$ represents the number of received symbols. A Raptor code has the property that the decoding success probability increases with each received additional symbol. Thus, the reception overhead of a Raptor code depends on $k$ and the desired probability that the source block can be fully reconstructed from the received symbol set [11]. 
Although Raptor codes impose an additional overhead, they are very attractive due to properties such as low complexity and flexibility. For example, the processing requirements for a Raptor code increase linearly with source block size $k$. These properties often allow Raptor codes to be implemented in software, which is uncommon for alternatives such as RS codes [15]. Furthermore, the number of source symbols $k$ and encoded symbols $n$ can be as large as desired. However, the standardized R10 code, which is a systematic version, has coding parameters of $4 \leq k \leq 8192$ and $k \leq n \leq 65,546$. The latest RQ can support up to 56,403 source symbols in a single source block and generate up to $2^{24}$ encoded symbols [12]. This property makes Raptor codes desirable for carousel-based services since the probability of receiving duplicate symbols can be significantly reduced.

RQ codes offer better coding efficiency (very close to ideal codes) compared with R10, which requires $k \geq 1000$ [13]. This allows the use of a flexible range of source block sizes, i.e. in practice, using small block sizes is better for devices with limited power and processing capability such as smartphones or tablets.

\subsection{Data carousel}

A data carousel, or broadcast disk, is a traditional way of providing reliable multicast servers over fixed networks. With a data carousel, the transmitter divides the file(s) into symbols, puts them into packets and then repeatedly transmits the packets in a cyclic fashion. Receivers may join the carousel at any time and normally leave once they have received all the packets that belong to a particular file(s). The elapsed time is called the file download time.

For file download delivery, error-free reception of the files is typically required. However, wireless communication channels are prone to errors (which result in packet loss) and, as a consequence, receivers may not obtain all the packets in a file, or set of files, in a single transmission cycle. Thus, it is necessary to wait for the next cycle for the chance to successfully retrieve the file, as shown in Fig. 1. In the traditional data carousel, for each file, the same set of packets is sent per cycle. This leads to the observation of duplicate packets at the receiver.

\section{FEC data carousel model}

A block AL-FEC code can be used in conjunction with a data carousel to improve its overall performance. The resulting system is referred to as a FEC data carousel [16]. In this case, in each carousel cycle, some repair symbols are sent alongside the original symbols. Although this approach improves the performance of the carousel, duplicate packets still occur at the receiver if the user needs more than one cycle to acquire the file(s). Therefore, in order to prevent duplicate packets at the receiver, we combine data carousels with Raptor fountain codes.

Our model can be summarised as follows. A file is divided into source blocks, with these blocks further divided into $k$ source symbols each of $T$ bytes $(B)$. A systematic RQ encoder is then applied to each individual source block of the file to generate the encoded data. The partitioning process and transmission schedule used in our RQ software is shown in Fig. 2. Each time, the RQ encoder generates a single encoded symbol from each source block of every file. As shown in Fig. 2, the first encoded symbol from each of the source blocks is sent, followed by the second and so on. In this case, symbols of each block and file are interleaved over time. As the code is systematic, the first $k$ encoded symbols are the source (original) symbols. RQ codes can be used to generate $2^{24}$ repair symbols from a source block, therefore the maximum number of repair symbols in the RQ software is set to $2^{24}$. Hence, each time a new encoded symbol is sent for each source block, duplicate packets are avoided at the receiver.

At the receiver, the Raptor decoder waits to collect all the user datagram protocol (UDP) packets belonging to a given source block. If the total number of received symbols for a block is $r=k+\varepsilon$, the Raptor decoder is successful and all source packets are recovered and delivered to the application layer. However, if the decoder fails, the receiver waits for more packets until successful decoding is possible.

In this work, one encoded symbol is placed into one UDP/internet protocol (IP) packet and hence the terms packet and symbol can be used interchangeably. Also, 802.11 multicast/broadcast packets cannot be fragmented

\begin{tabular}{|l|l|l|l|l|l|l|l|l|l|l|l|}
\hline File A is not fully retrieved \\
Fig. 1 Example of a data carousel
\end{tabular}




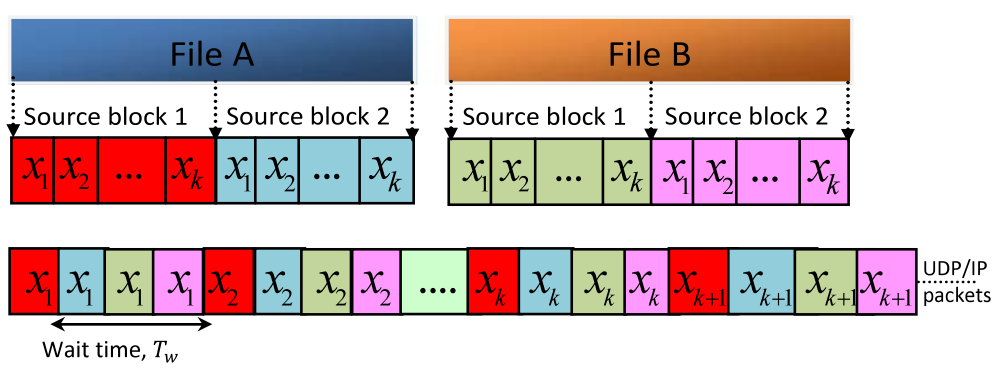

Fig. 2 Interleaved FEC data carousel model

at the MAC layer, resulting in a 1:1 mapping between encoded symbol and PHY layer protocol data units (PPDU).

\section{Problem formulation}

The average download time is defined as the time elapsed between a user joining the carousel and then receiving sufficient packets to reconstruct the file. This is a key metric and reflects the overall system performance (service quality) for download delivery in multicast networks. The objective is to reduce the average download time and hence increase the user quality of experience (QoE).

For simplicity, in the analytical model, we assume that the transmitter sends a fixed set of files in a cyclic fashion. Each file has the same size ( $S$ bytes) and consists of $N_{\mathrm{SB}}$ source blocks as the source block size $k$ and symbol size $T$ are fixed for each file, hence $N_{\mathrm{SB}}=\left[\frac{S}{k \cdot T}\right]$.

When fountain codes are implemented in the data carousel system, each of the received symbols will be different and useful for decoding. This is unlike traditional block codes where the same set of data is transmitted in each cycle, requiring the calculation of the number of unique received symbols in each cycle [5]. However, when Raptor codes are implemented, there is no such constraint so the number of received symbols/packets depends only on the packet error rate (PER), $p$. Based on this, next we formulate an estimate of the download time for the carousel system when combined with fountain codes.

The download time for a file depends on the PER in the source blocks, which is a function of the mean channel signal-to-noise ratio (SNR) $s$, the modulation and coding scheme (MCS) $m$, the source block size $k$ and the symbol size $T, p_{l}(s, m, k, T), l=1, \ldots, N_{\mathrm{SB}}$. As each Raptor block in a file is decoded separately, the source block with the highest PER defines the download time. This occurs since the block with the highest PER must wait the longest time at the receiver to collect sufficient packets to enable its decoding, and hence to gain access to the entire file.
Therefore, we first define the source block SB that requires the highest number of packets $N$ for successful decoding (i.e. the last decoded source block) as follows:

$$
\begin{aligned}
& \mathrm{SB}=\underset{l}{\arg \max }\left(p_{l}(s, m, k, T)\right) \\
& N(s, m, k, T)=\max _{l}\left[\left(\frac{k+\varepsilon}{1-p_{l}(s, m, k, T)}\right)\right] .
\end{aligned}
$$

These PER values are obtained from a detailed PHYMAC layer simulator as described in Section 5.1.2. Then, we define the download time $T_{D}$ of a file for any MCS $m$ and for Raptor code parameters $k$ and $T$ as follows:

$$
\begin{aligned}
T_{\mathrm{D}}(s, m, k, T)= & \left(N_{\mathrm{SB}} \cdot N_{F} \cdot(N(s, m, k, T)-1)+\mathrm{SB}\right) T x\left(m, L_{\mathrm{PSDU}}\right) \\
+ & T_{\mathrm{w}} \\
T x\left(m, L_{\mathrm{PSDU}}\right)= & \mathrm{DIFS}+T_{\mathrm{BO}}+T_{\mathrm{PREAMBLE}} \\
& +T_{\mathrm{SYM}} \cdot N_{\mathrm{SYM}}+\mathrm{SIFS} \\
N_{\mathrm{SYM}}\left(L_{\mathrm{PSDU}}\right)= & {\left[\frac{L_{\mathrm{PSDU}}+2.75}{N_{\mathrm{DBPS}}^{m}}\right] }
\end{aligned}
$$

$N_{F}$ is the total number of files in the carousel and $T x$ is the time required to transmit a PHY layer PPDU, which is the sum of the time required to transmit the preamble, the protocol service data unit (PSDU) $L_{\mathrm{PSDU}}$ and the distributed inter-frame spacing (DIFS), the short frame spacing (SIFS) and the back-off time $T_{\mathrm{BO}}$. All parameters follow the $802.11 \mathrm{n}$ standard in [17]. $N_{\mathrm{SYM}}$ is the number of orthogonal frequency-division multiplexing (OFDM) symbols required for transmission of an $L_{\text {PSDU, }}$ and $T_{\text {SYM }}$ is the OFDM symbol duration. The addition of 2.75 in (5) covers the overhead associated with the service and tail bits. $N_{\text {DBPS }}$ is the number of data bytes per OFDM symbol for a given MCS mode $m$. $L_{\text {PSDU }}$ is the sum of the Raptor symbol size $T$ and the total number of higher layer headers $\left(L_{\mathrm{hdr}}=64 \mathrm{~B}\right)$, which consists of an 8-B UDP header, 20-B IP header and 36-B MAC header. $L_{\mathrm{PSDU}}=T+L_{\mathrm{hdr}}$. $T_{\mathrm{w}}$ represents the wait time between a user joining the carousel and the start of the file download. In this work, the number of files in 
the carousel is equal to $1\left(N_{F}=1\right)$, therefore the wait time is $T_{\mathrm{w}}=0$.

The main benefit of the interleaved carousel model is to randomise the PER (which prevents burst errors) amongst the source blocks and therefore the mean and variance of the PER over all source blocks are very close. This means that the number of required packets per source block of a file is very close, and as a result, the download time is much lower compared to sequential transmission [18]. To explain this in more detail, the mean and variance of the PER is plotted for each source block of a file, which consists of 30 source blocks as shown in Fig. 3 for the sequential and interleaved carousel models using packet loss traces obtained via the measurements described in our previous work [18]. In the interleaved model, the maximum observed PER is 0.042 , whereas in the sequential model, it is 0.17 . These PER values define the download time for that file since in order to decode the file, all the source blocks must be decoded successfully. Clearly, the sequential model needs more time to retrieve sufficient packets for that source block. Therefore, for the same mean PER, the download time depends solely on the maximum source block PER.

\section{Evaluation methodology}

The performance of the proposed system was assessed using an advanced multi-layered simulator and through a campaign of real-world measurements. The main metric for system evaluation was the average download time for a test file. Measurements and simulations were performed several times with an averaged taken over all realisations. In order to optimise the end-to-end system performance and provide higher QoE to the users, it is crucial to define the system parameters that affect average download time. By building a practical implementation, we are able to investigate these parameters in real-world environments using representative hardware. The detailed information on the simulation and measurement configuration are given in the following sections.

\subsection{Simulation setup}

In order to reduce computational complexity, the overall system is divided into modular subsystems (channel, Wi-Fi PHY-MAC layer and FEC data carousel simulator), each of which is modelled independently. Since the FEC data carousel model was explained in Section 3, only the channel and Wi-Fi PHY-MAC layer models are explained in the following subsections.

\subsubsection{Channel model}

A state-of-the-art outdoor 3D ray tracer [19] was used to model the time-varying channel matrix $\mathbf{H}$ between the access point (AP) and each user equipment (UE). The ray tracer makes use of the physical laws of radiowave propagation such as reflection, diffraction and scattering and identifies all significant ray paths between the $\mathrm{AP}$ and the UE in $3 \mathrm{D}$ space. The ray tracing database had a resolution of $2 \mathrm{~m}$ and included buildings, foliage and terrain data in the area. Point-source ray tracing was conducted from the user to the AP to provide information on the amplitude, phase, time delay, angle of departure (AoD) and angle of arrival (AoA) of each multipath component (MPC).

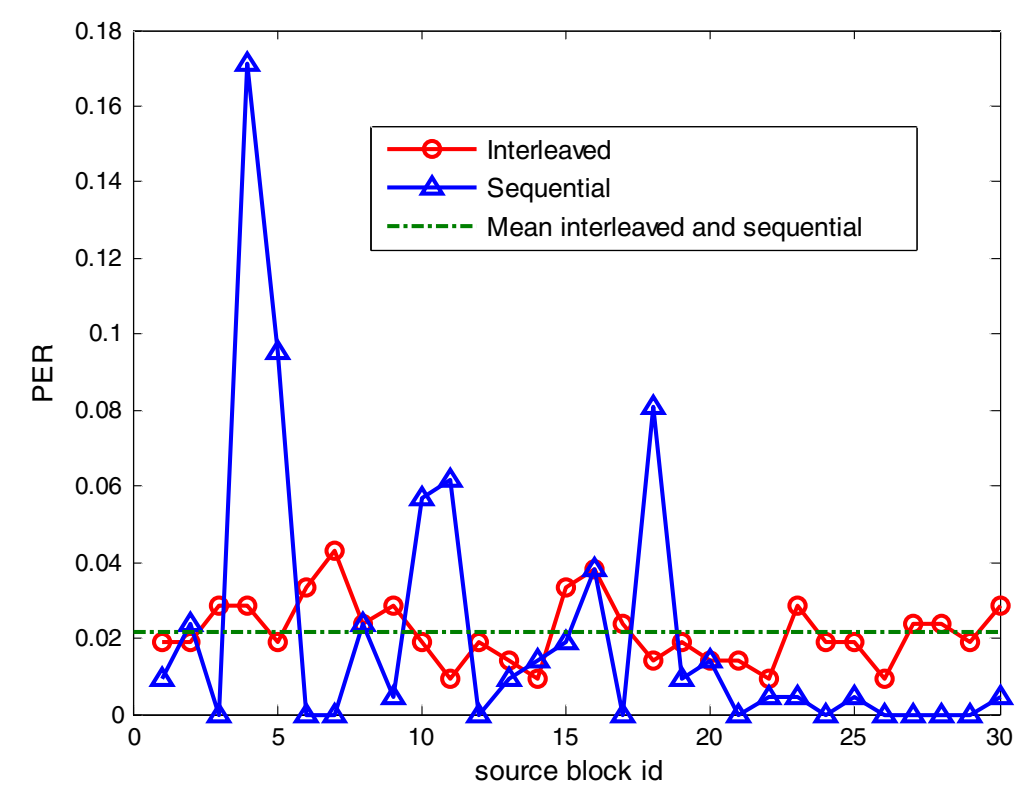

Fig. 3 PER in each source block for sequential and interleaved carousel models, MCS 2 


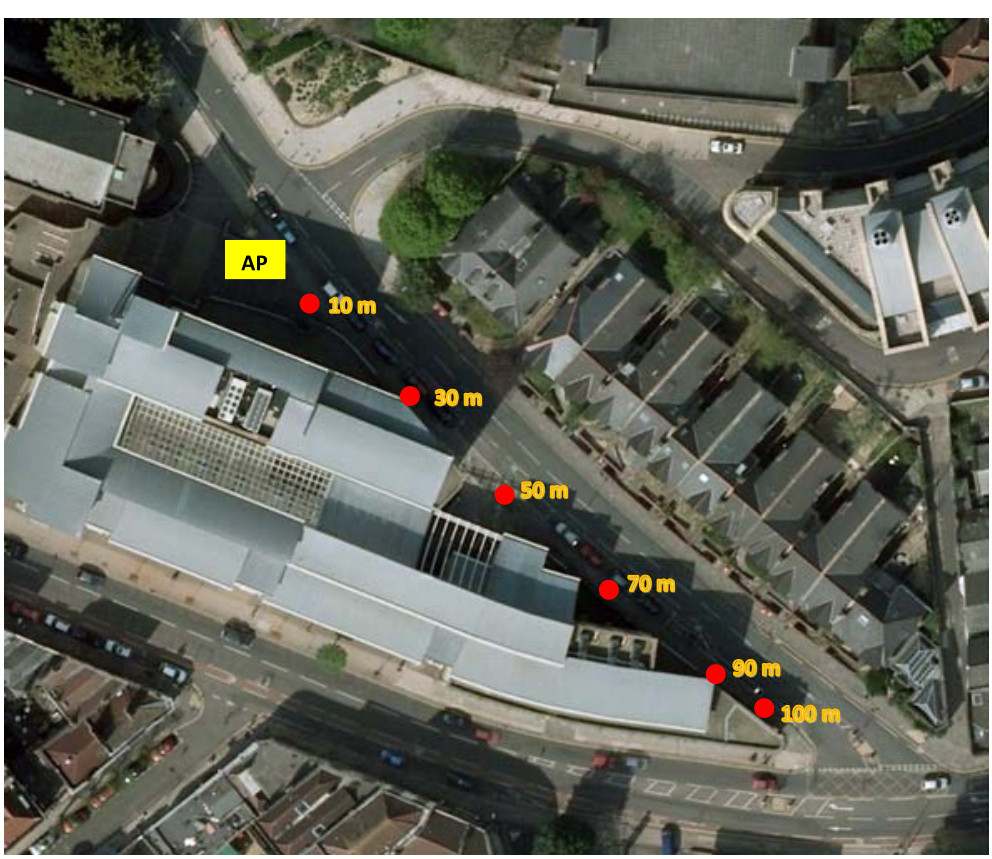

Fig. 4 Locations of AP and UES

The theoretic Wi-Fi performance was evaluated in our trial location using a geographic model of Bristol as shown in Fig. 4. The users were served by a single AP operating in the $2.4-\mathrm{GHz}$ band. The AP was mounted $2.5 \mathrm{~m}$ above ground level. The elevation of the user antennas was $1 \mathrm{~m}$ above ground level. The system was modelled based on measured element patterns from a Netgear 7000 AP and a Toshiba Android tablet. The tablet had a single antenna while the AP made use of three antennas. The radiation patterns of the AP antennas and the UE antenna were measured in the University of Bristol's anechoic chamber. All patterns were measured in $3 \mathrm{D}$ and included phase and polarisation information.

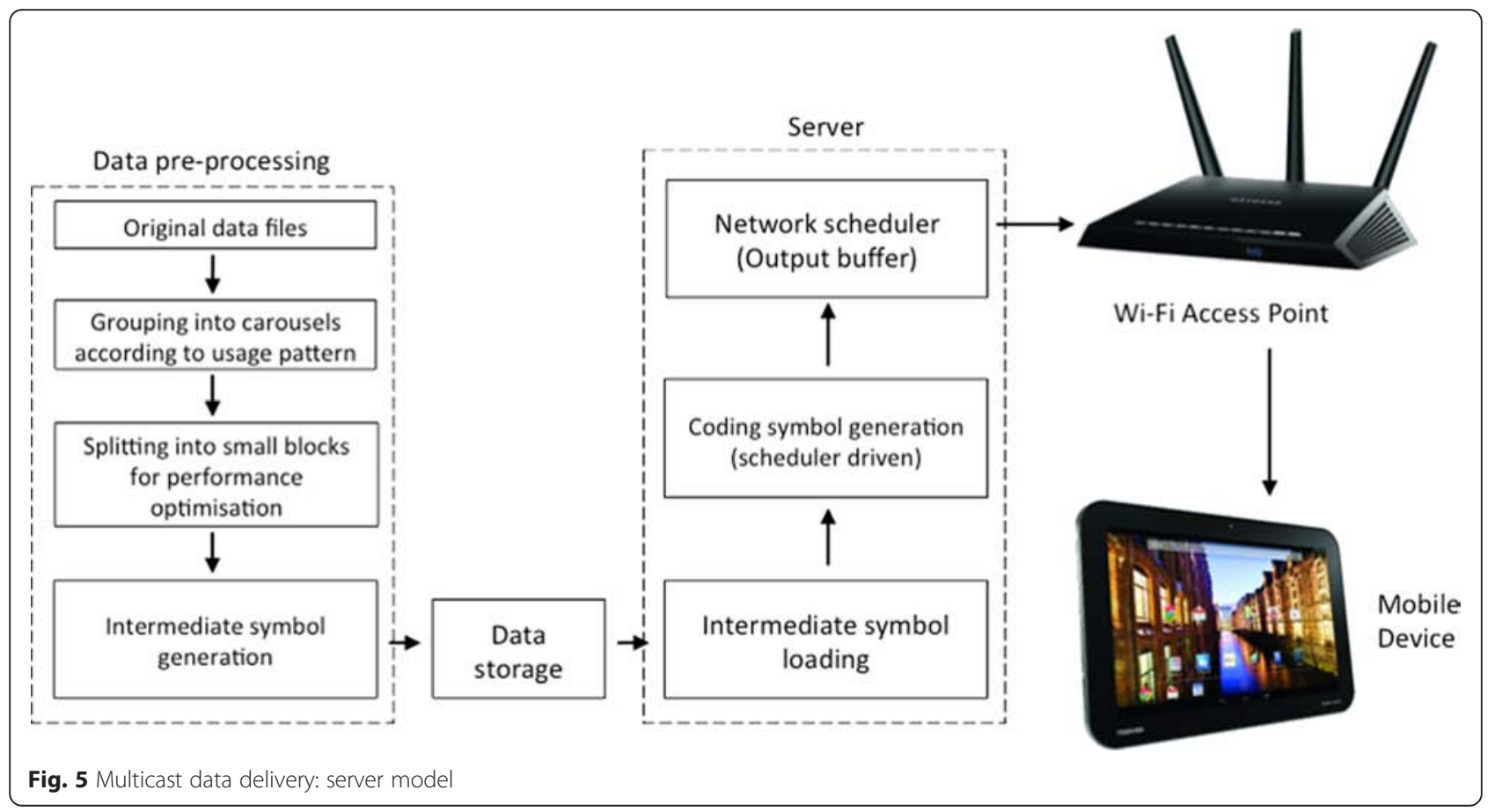



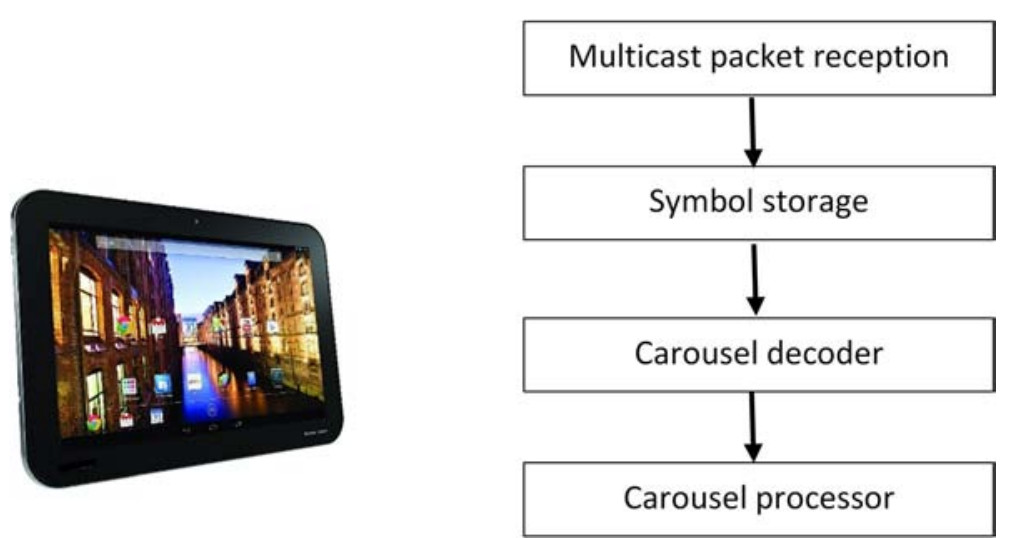

Fig. 6 Multicast data delivery: client model

\subsubsection{PHY (link-level abstraction) and MAC model}

Performing bit accurate PHY simulations for large numbers of parameter sets is computationally prohibitive. Therefore, an effective SNR mapping (ESM) PHY abstraction model, known as the received bit mutual information rate (RBIR) [19] technique, is used to estimate the PER statistics. In the ESM method, a block of OFDM sub-carrier SNRs, which varies due to frequency selective fading, is transformed into a single effective SNR (ESNR) value using (6).

$$
\mathrm{ESNR}=\Phi_{m}^{-1}\left\{\frac{1}{N_{\mathrm{SC}} \cdot N_{\mathrm{ss}}} \sum_{n=1}^{N_{\mathrm{SC}}} \sum_{k=1}^{N_{\mathrm{sS}}} \Phi\left(\mathrm{SNR}_{n, k}\right)\right\}
$$

In (6), $\mathrm{SNR}_{n, k}$ represents the post-processing SNR for the $k$ th spatial stream of the $n$th sub-carrier and $m$ represents the modulation order. $N_{\mathrm{SC}}$ represents the number of sub-carriers in the block, $N_{\mathrm{ss}}$ is the maximum number of spatial streams and $\Phi(\bullet)$ is an invertible function. The mutual information (MI) ESM approach is used in this paper. This defines $\Phi(\bullet)$ as the symbol information (SI) as given in (7),

$$
\operatorname{SI}(\gamma, m)=E_{x y}\left\{\log _{2} \frac{P(Y \mid X, \gamma)}{\sum_{X} P(X) P(Y \mid X, \gamma)}\right\},
$$

where $Y$ denotes the received symbol with input SNR equal to $\gamma$ and $P(Y \mid X, \gamma)$ is the additive white Gaussian noise (AWGN) channel transition probability density conditioned on the noise-free transmit symbol $X$.

This ESNR value is then used to define the instantaneous PER for any MCS mode using a non-faded PER versus SNR look-up table. This table is generated using a bit accurate Wi-Fi simulation for an AWGN channel. By generating a set of instantaneous and uncorrelated channels (typically 1000 snapshots) for each point-to-point link, the PER can be calculated per channel as above (taking into account the frequency selective fading and the Rican K-factor) and then averaged to generate the mean PER in the fading channel. The ESM PHY abstraction method is described in greater detail in [20].

The MAC layer model, which uses the MAC distributed coordination function (DCF) with basic access as defined in the IEEE 802.11 standard [1], models the

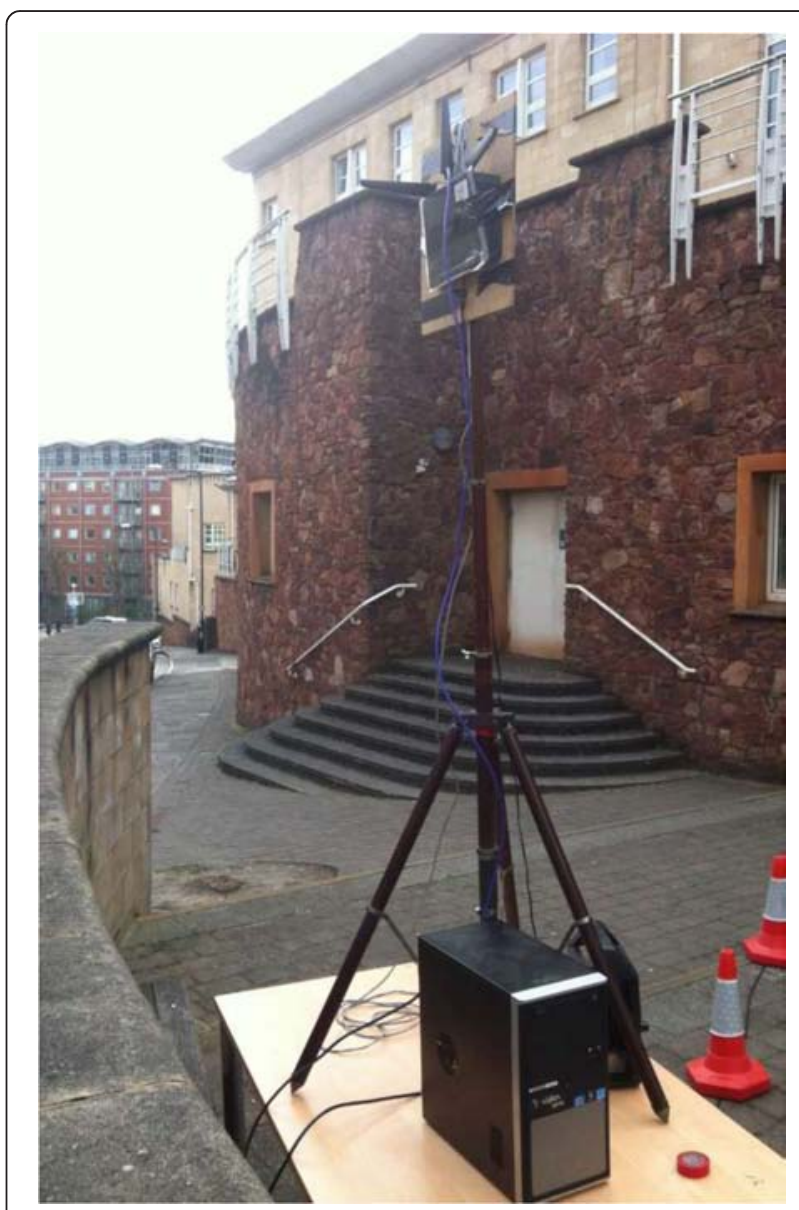

Fig. 7 Measurement scenario 
Table $1802.11 \mathrm{n}$ transmission modes

\begin{tabular}{|c|c|c|c|c|c|c|}
\hline \multirow[t]{2}{*}{ MCS index } & \multirow[t]{2}{*}{ Modulation } & \multirow[t]{2}{*}{ Coding rate } & \multirow[t]{2}{*}{$N_{D B P S}$} & \multirow[t]{2}{*}{ Data rate $(\mathrm{Mbps}), R_{\mathrm{D}}$} & \multicolumn{2}{|c|}{ Multicast server rate (Mbps), $R_{\mathrm{M}}$} \\
\hline & & & & & High & Low \\
\hline 0 & BPSK & $1 / 2$ & 3.25 & 6.5 & 4 & 2 \\
\hline 1 & QPSK & $1 / 2$ & 6.5 & 13 & 8 & 4 \\
\hline 2 & QPSK & $3 / 4$ & 9.75 & 19.5 & 12 & 6 \\
\hline 3 & 16-QAM & $1 / 2$ & 13 & 26 & 16 & 8 \\
\hline 4 & 16-QAM & $3 / 4$ & 19.5 & 39 & 24 & 12 \\
\hline 5 & 64-QAM & $2 / 3$ & 26 & 52 & 32 & 16 \\
\hline 6 & 64-QAM & $3 / 4$ & 29.25 & 58.5 & 40 & 20 \\
\hline 7 & 64-QAM & $5 / 6$ & 32.5 & 65 & 40 & 20 \\
\hline
\end{tabular}

Abbreviations: BPSK binary phase-shift keying, QPSK quadrature phase-shift keying, QAM quadrature amplitute modulation

packet loss pattern for a sequence of packets. Since data is sent as a broadcast stream to multiple users, ARQ is not implemented.

\subsection{Measurement setup}

Measurements were performed in order to investigate system performance in a real-world environment using a practical hardware and software. These measurements help validate our simulation results and show where practical performance deviates from theory and simulation. To this end, we developed a server and client model that supports multicast multimedia data delivery to Android tablets via a Wi-Fi access point.

\subsubsection{Server model}

The server was responsible for generating the Raptor FEC packets from the carousel data files and is also used to send the resulting data stream via UDP broadcast for wireless transmission. The RQ software, which runs in real-time on a desktop PC (encoder) and multiple Android tablets (decoders), has been developed by the authors based on the RFC 6330 standard as explained in [21]. Basically, the RQ encoding process consists of three steps: (1) the creation of an $L \times L$ encoding matrix A (for a specific block size $k$ ), (2) the generation of $L$ intermediate symbols by multiplying the original data

Table 2 System parameters

\begin{tabular}{ll}
\hline Parameters & Values \\
\hline Source block size, $k$ & $50,200,400$ \\
Raptor symbol size, $T$ & $500,1000,1400 \mathrm{~B}$ \\
Number of files in the carousel, $N_{F}$ & 1 \\
File size, $S$ & $5.2 \mathrm{MB}$ \\
DIFS & $34 \mu \mathrm{s}$ \\
SIFS & $16 \mu \mathrm{s}$ \\
OFDM symbol duration, $T_{\text {SYM }}$ & $4 \mu \mathrm{s}$ \\
PLCP preamble time, $T_{\text {PREAMBLE }}$ & $40 \mu \mathrm{s}$ \\
\hline
\end{tabular}

Abbreviation: $P L C P$ physical layer convergence protocol with the encoding matrix, and (3) the generation of the encoding symbols by combining a small number of intermediate symbols. The second step was optimised by pre-multiplying the original data with the encoding matrix and storing the intermediate symbols on the server's hard drive. Figure 5 summarises the transmission process of the multicast data delivery system. First, the original files are grouped into carousels depending on the usage pattern (there would be more than one

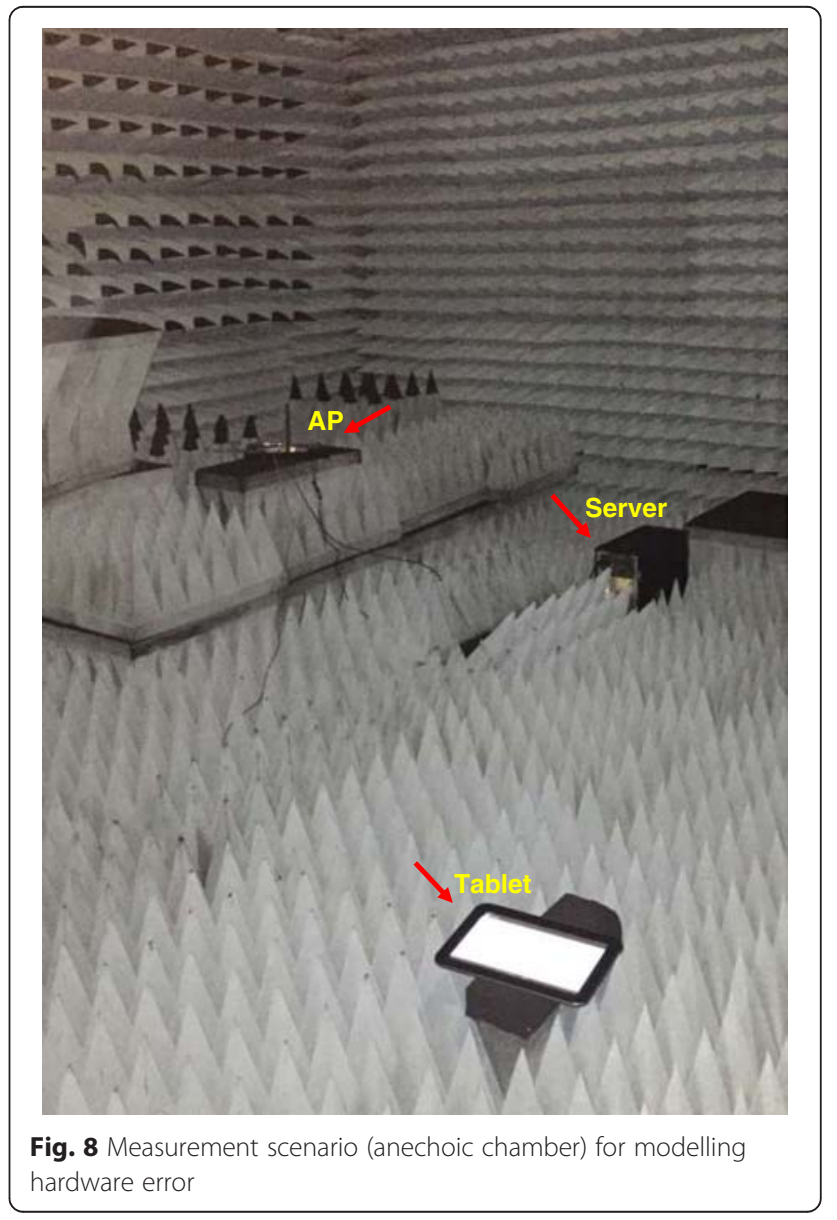


carousel). Then, the files are divided into equal small blocks except for the final block in the RQ encoding process.

\subsubsection{Client model}

Toshiba AT10LE Android tablets were used as our test clients. These have a single embedded antenna and connected to the AP at $2.4 \mathrm{GHz}$. The client software was developed in this project to intercept the multicast Wi-Fi stream, apply application layer decoding and write the multimedia data files to internal memory.

Figure 6 shows the process at the client. It is seen that at the client, the received multicast packets are stored. If the number of received packets is $r \geq k^{\prime}$, where $k^{\prime}$ is the number of source plus padding symbols in an extended source block [21], then RQ decoding is implemented over these symbols to recover the source block of the file. The first part of the decoding is the creation of an $L \times L$ encoding matrix $\mathbf{A}$ and then the generation of $L$ intermediate symbols by multiplying the received data with the encoding matrix. Finally, the $k$ source symbols are regenerated by employing LT decoding on intermediate symbols.

\subsubsection{Measurement scenario}

The measurements were conducted at the University of Bristol using two Toshiba tablets and a single Netgear 7000 AP. The Netgear unit was flashed with a development software image provided by Broadcom to allow access to MAC/PHY level parameters and also for the configuration of modes such as the multicast MCS rates.

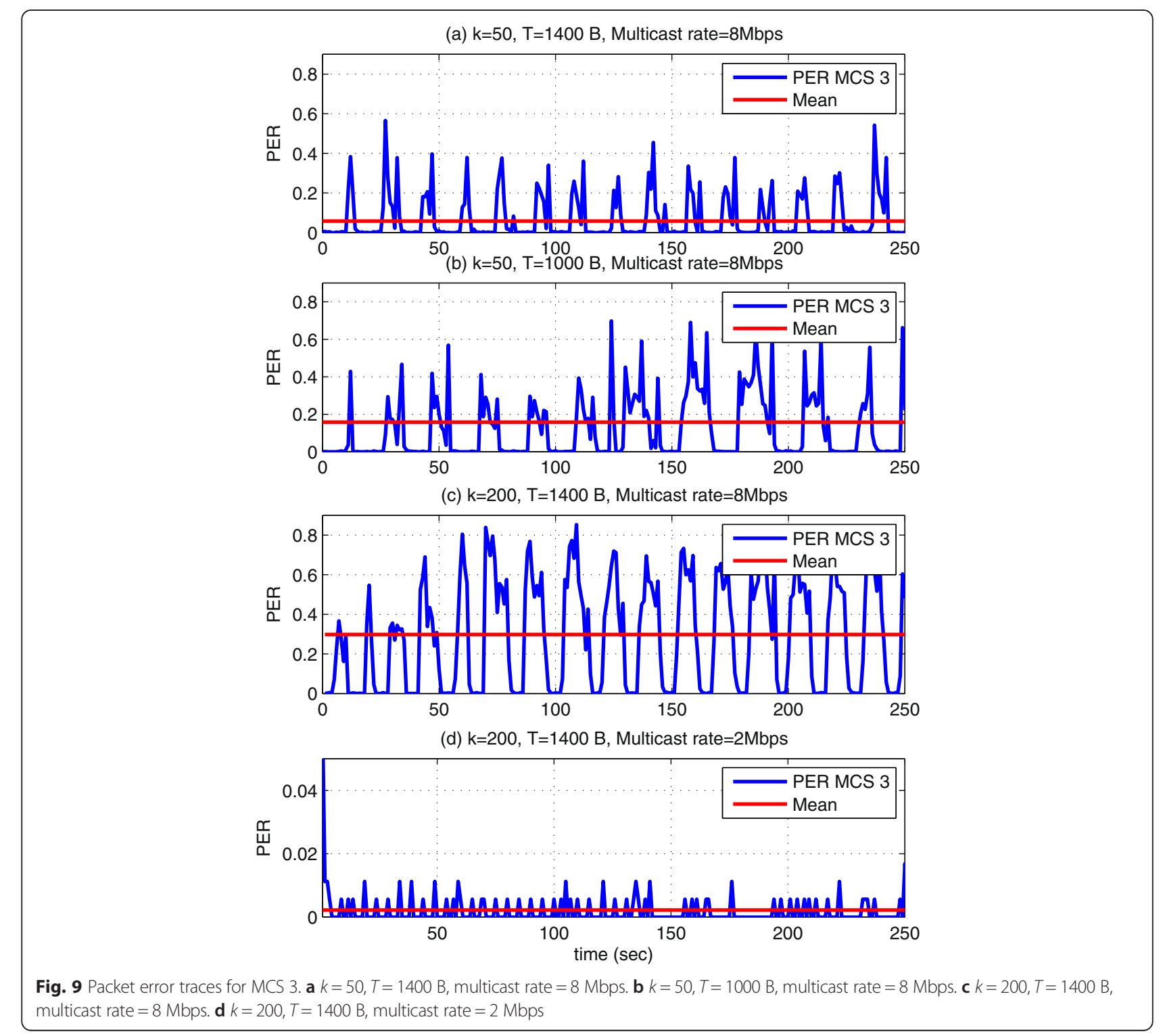


Figure 7 shows the measurement scenario. The server was located on a table and was connected to the Wi-Fi AP, which was mounted on a tripod, via an Ethernet cable. The AP height was set at $2.5 \mathrm{~m}$ above the ground level (i.e. the same as in the simulations). The measurements were performed at different locations (distances) from the AP in Woodland Road, Bristol. During the measurement runs, the users stood on average for $2 \mathrm{~min}$ at each point. Data in the form of average download time, received signal strength indicator (RSSI), AP-totablet separation distance and packet loss traces were logged for each location and parameter set.

\section{Evaluation parameters}

This section gives detailed information on the parameters used in the measurements and simulations. There are many parameters that affect the user QoE. During all the tests (measurements and simulations), it was assumed that the number of files in the carousel was 1 . The size of the file was fixed at 5.2 MB, which is typical for multimedia content such as a music file or short video clip for a mobile device [22]. Given these assumptions, we evaluated the MCS mode and Raptor code parameters, source block size $k$ and symbol size $T$, which have a significant impact on the download time. Depending on the tablets distance from the AP, large variations can be seen in the received power and hence the Wi-Fi packet loss rate [23].

The transmission modes used in our tests assume an 802.11n $20-\mathrm{MHz}$ channel profile with an $800-\mathrm{ns}$ guard interval (GI) [17]. The multicast server rates (or multicast/application data rates) are shown along with the transmission modes in Table 1.

In practice, the peak application data rates are lower than the PHY data rates due to channel access delays, upper layer overheads and/or hardware bottlenecks and driver restrictions [24, 25]. Therefore, in order to prevent transmission of data at rates higher than the mobile device is capable of receiving (the tablets dropped packets if they arrived too rapidly), we set the multicast server rate in Table 1 to be much lower than the peak PHY rate [26]. We define two multicast data rates, namely high and low in Table 1, in order to investigate the end user performance in terms of download time with respect to different multicast server rates. Furthermore, Table 2 details the system parameters used in the PHY layer, data carousel and Raptor encoder/decoder.

\section{Results and analysis}

Packet loss can occur for a number of reasons, for example deep fading, hardware and driver limitations, network congestion and competing interference. To the best of the authors' knowledge, no current simulations consider the impact of hardware platform and driver limitations in their Wi-Fi multicast studies. In order to make our Wi-Fi multicast simulations to tablets more realistic, we model the error patterns originating from the client in ideal channel conditions and incorporate these into the simulator. In this section, we validate our simulation results and evaluate the system performance for different PHY and application layer parameters.

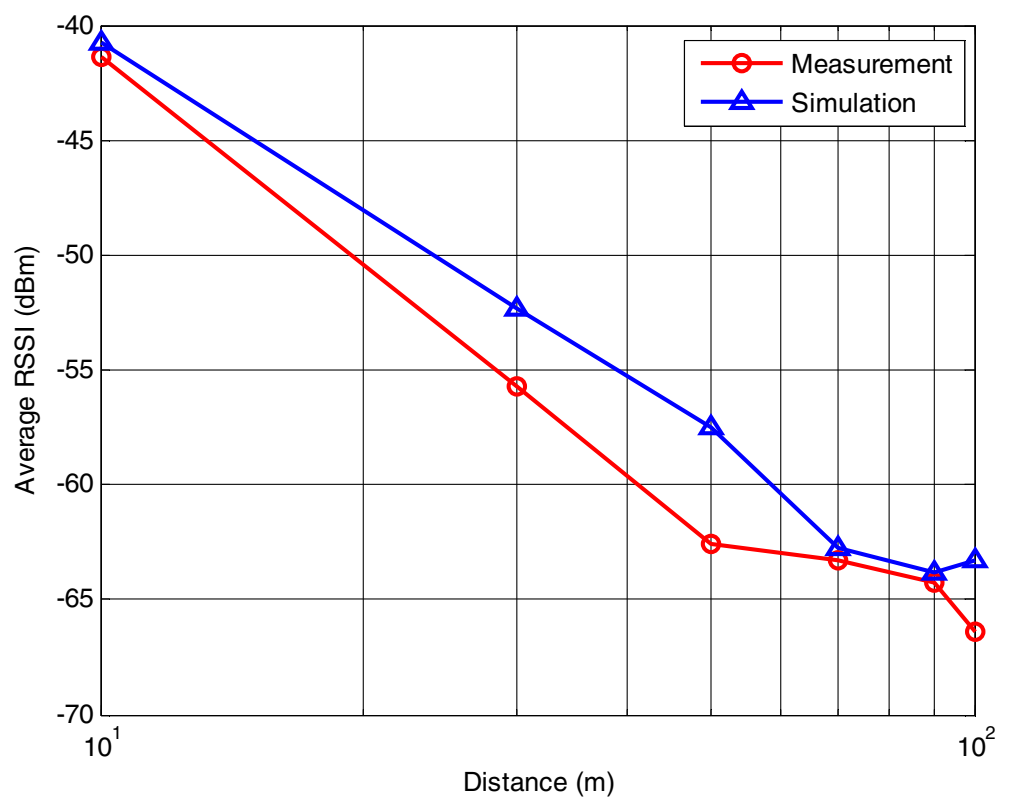

Fig. 10 Comparison of average RSSI 


\subsection{Modelling hardware errors}

In order to model the packet loss introduced by hardware and software limitations on constrained host platforms (cpu, bus, memory), where the incoming packet rate exceeds the devices' ability to consume the traffic, we first need to eliminate all other sources of packet loss at the tablet. To that end, we performed experiments in an anechoic chamber as shown in Fig. 8. Inside the chamber, there is no interference and no multipath (effectively an AWGN channel). Furthermore, the received signal level at the client is excellent since it was located within a couple of metres of the access point. We placed the Wi-Fi multicast server, AP and a single tablet inside the chamber and logged data over 5 min durations for each parameter set. Measurements were performed for different PHY layer and application layer parameters in order to identify their effects on the hardware errors seen in the tablet.

Figure 9 shows the received packet traces at the application layer for MCS 3 with different Raptor code

(a) Simulation

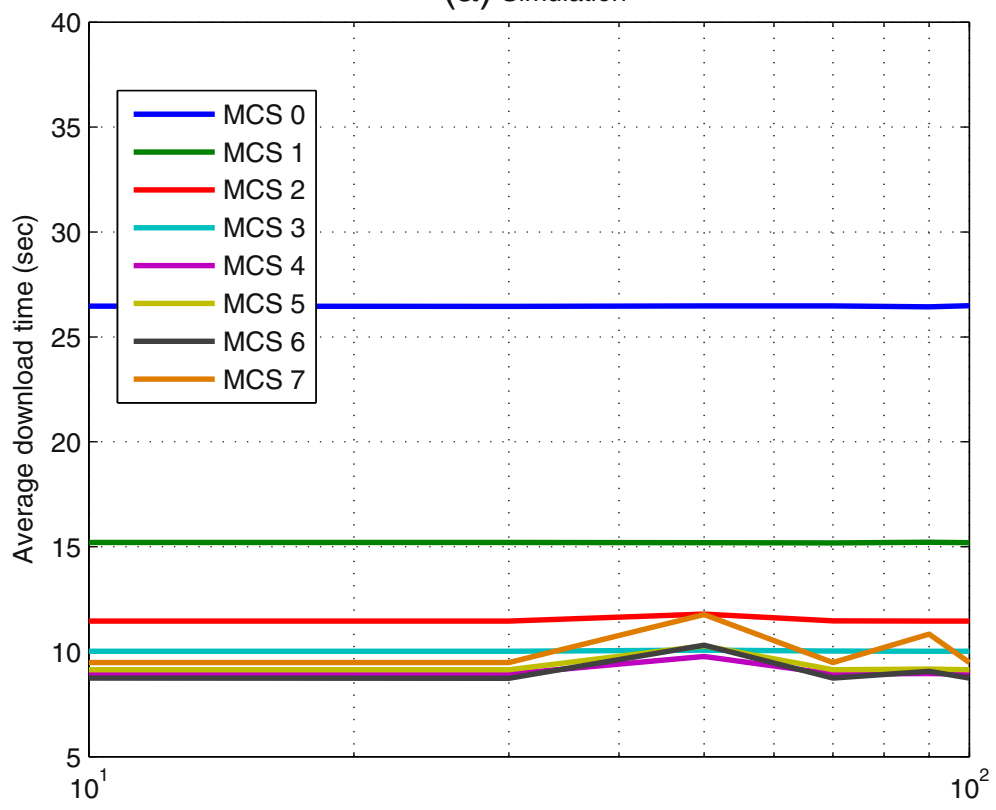

(b) Measurement

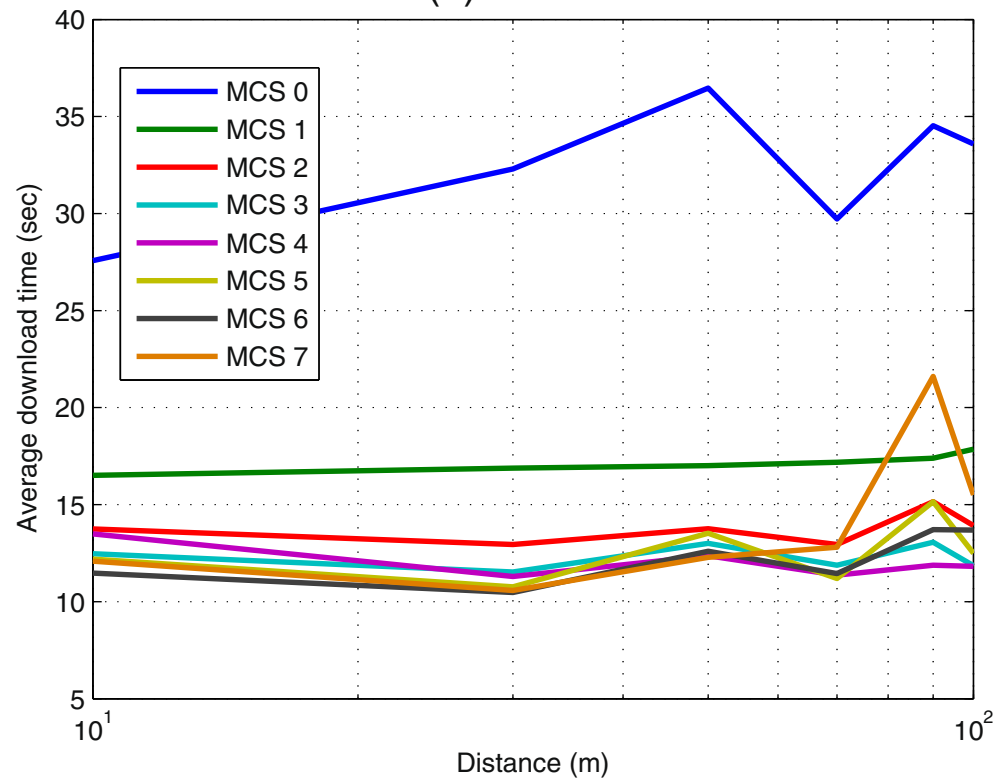

Fig. 11 Comparison of average download time, $k=50, T=1400 \mathrm{~B}$, lower multicast data rate. a Simulation. b Measurement 
parameters and multicast server data rates. It should be noted that measurements were performed for all MCS modes and for a range of multicast server rates using the Raptor parameters reported in Tables 1 and 2. It can be seen that the error patterns depend on the multicast server rate, the source block length and the symbol size. For the same source block size and multicast server rate, reducing the source symbol size results in higher PER (Fig. 9a, b), whereas for the same source block size and multicast data rate, reducing the source block size provides lower PER
(Fig. 9a, c). Furthermore, for the same Raptor code parameters, reducing the multicast server rate significantly reduces the PER at the application layer (Fig. 9c, d). These results reveal that the tablet has limited processing capability; therefore, it drops packets as the number of transmitted packets/second increases (for a given modulation and coding scheme, the transmitted packets per second increases as the multicast server rate increases and the source symbol size reduces). Moreover, the Raptor code processing overhead increases with increased source block size.

(a) Simulation

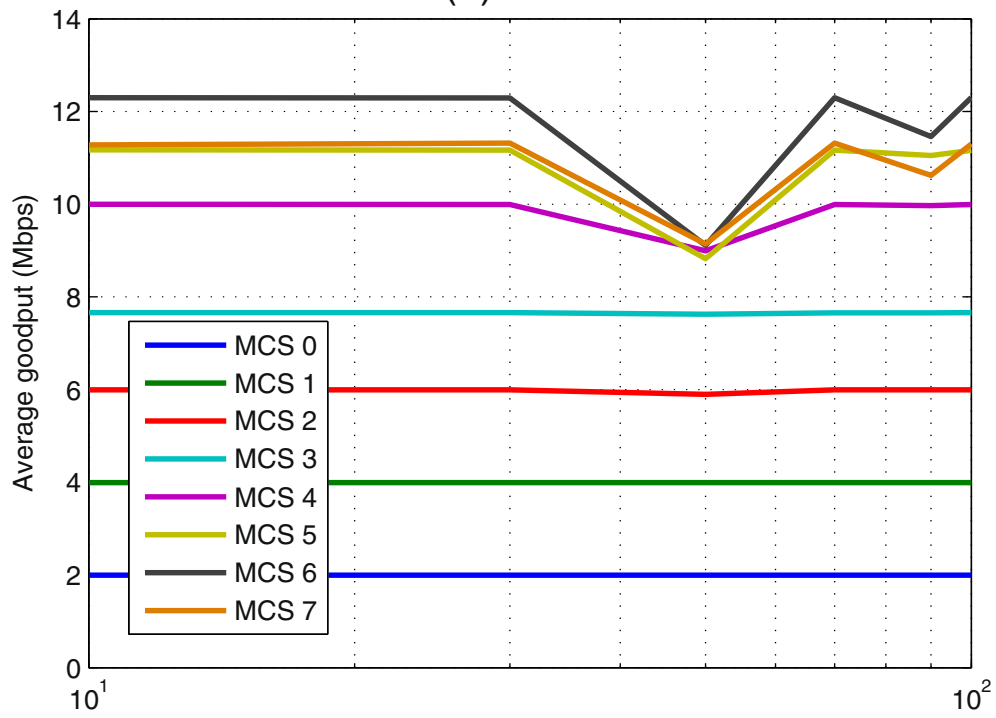

(b) Measurement

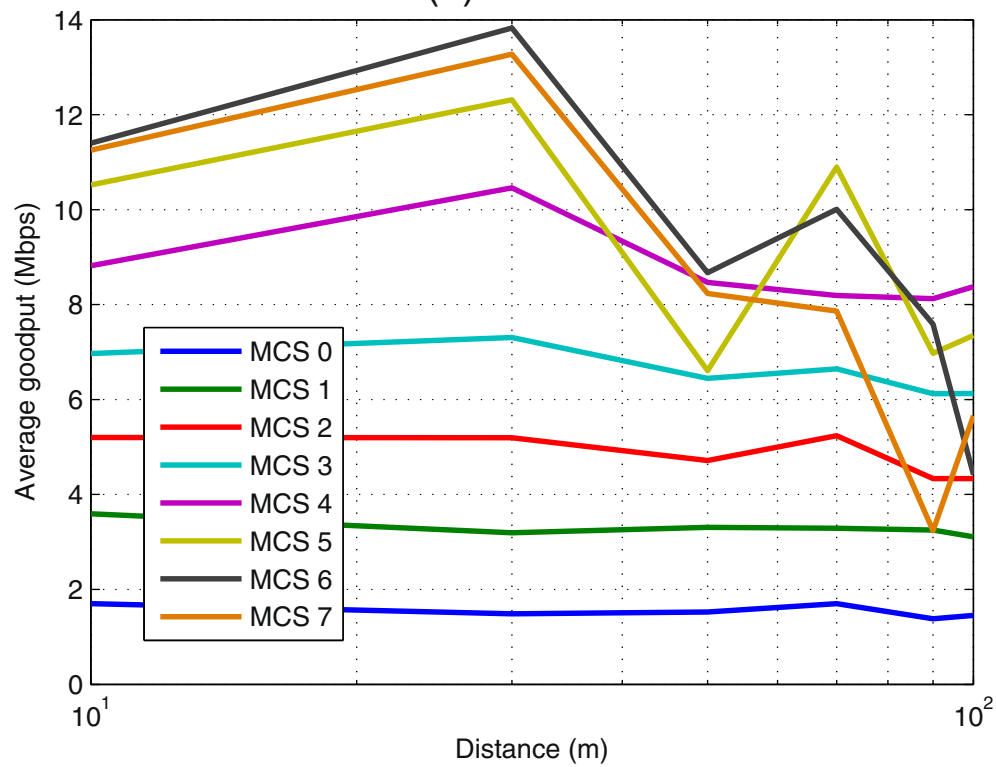

Fig. 12 Comparison of average goodput, $k=50, T=1400 \mathrm{~B}$, lower multicast data rate. a Simulation. b Measurement 


\subsection{Comparison of simulation and measurement results} After recording hardware packet loss patterns for each parameter set, we use these patterns in the simulator when calculating the average download time. In this experiment, the results are given for $k=50, T=1400 \mathrm{~B}$ and the lower of the two multicast server rate. Figure 10 shows predicted and measured RSSI values at each location in the trial environment. It can be seen that the ray tracer slightly overpredicts the received RSSI values. We believe that the difference between the RSSI value and the predictions comes from the mismatch between the tablet antenna rotations in the real measurements and the simulations (we do not capture the exact tablet orientation). As we compare the average download time, it can be seen in Fig. 11 that the predicted results are lower than the measured results. This is because the received power is very high, and as a result, the $\mathrm{PER}=0$. This is especially visible at lower MCS modes since these modes require less received power for reliable operation. Furthermore, the hardware errors are negligible for these modes (less than $5 \%$ ).

We also compare the attained goodput at the application layer before Raptor decoding (Fig. 12). The goodput $G$ is calculated as $G=(1-\mathrm{PER}) R_{\mathrm{M}}$, where $R_{\mathrm{M}}$ represents

(a) Low multicast rate

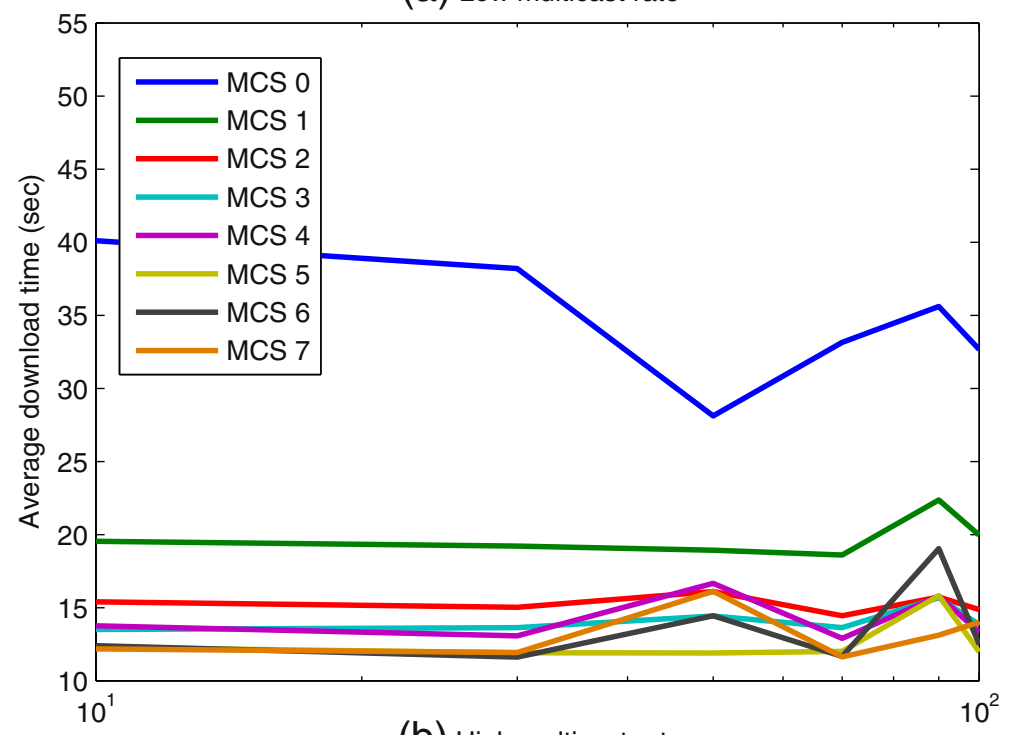

(b) High multicast rate

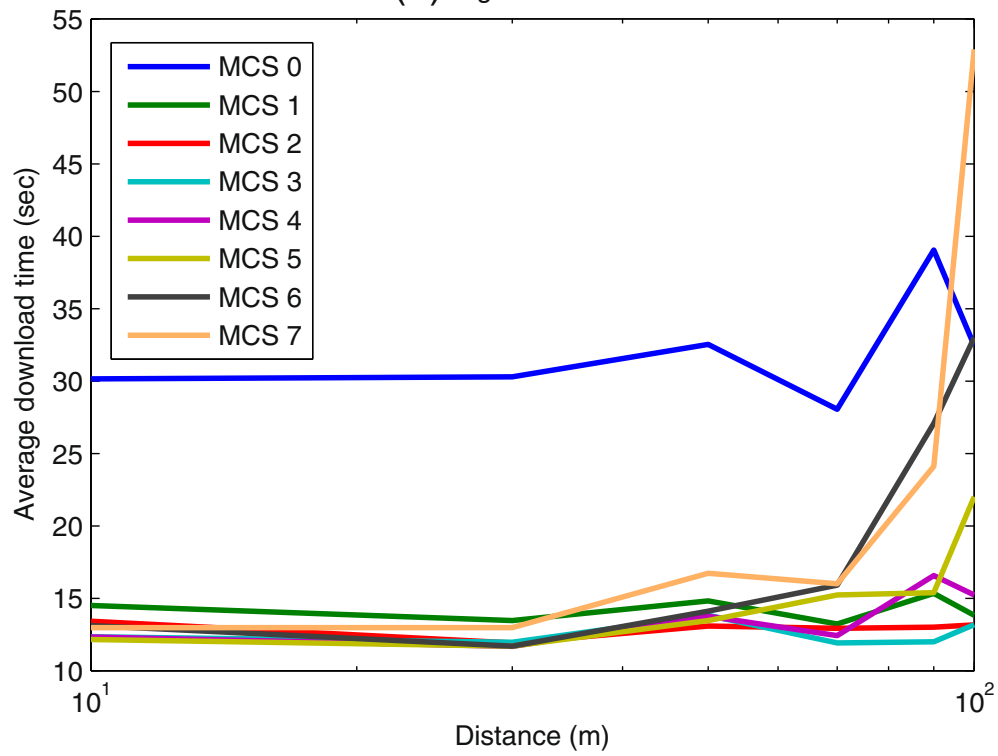

Fig. 13 Average download time versus distance, $k=200, T=1400$ B. a Low multicast data rate. b High multicast data rate 
the peak multicast transmission rate. As expected, since $\mathrm{PER}=0$, the goodput is equal to the multicast server rate for the lower modes. For the higher MCS modes, the goodput depends on the hardware error applied in the simulations. In the tablet measurements, the goodput is slightly lower than the multicast server rate. Differences between the simulator and the measurements could be due to real-world interference, since this is not included in the model.

In general, it is seen that the simulator results are consistent with the measurements. Therefore, in the following results, we limit our graphs to show the measured data.

\subsection{Analysis of multicast data rate}

In this experiment, we compare the average download time with respect to the multicast data rate for each MCS mode. Figure 13a, b shows the measurement results for the low and high multicast server rate, respectively. It can be seen that for lower/robust MCS modes, using higher multicast server rates provides lower download times since the PER is low as shown in Fig. 14. For higher MCS modes, using higher multicast server rates results in higher PER and, as a result, longer download times (especially at increased AP-to-tablet separation distances). Therefore, it is beneficial to use lower multicast server rates along with higher MCS

(a) Low multicast rate

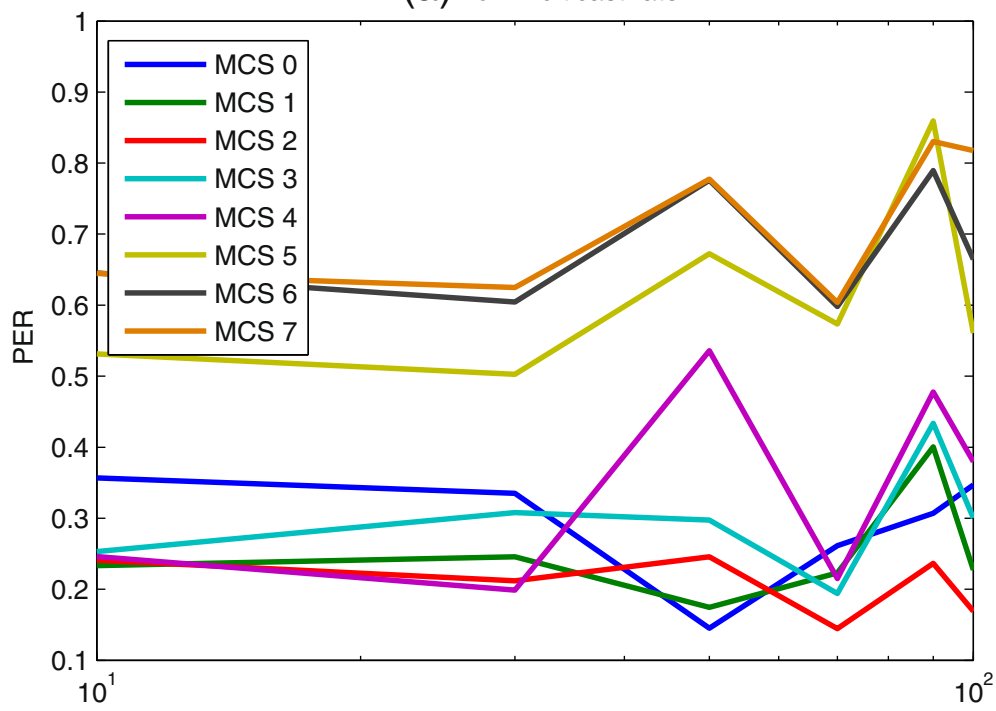

(b) High multicast rate

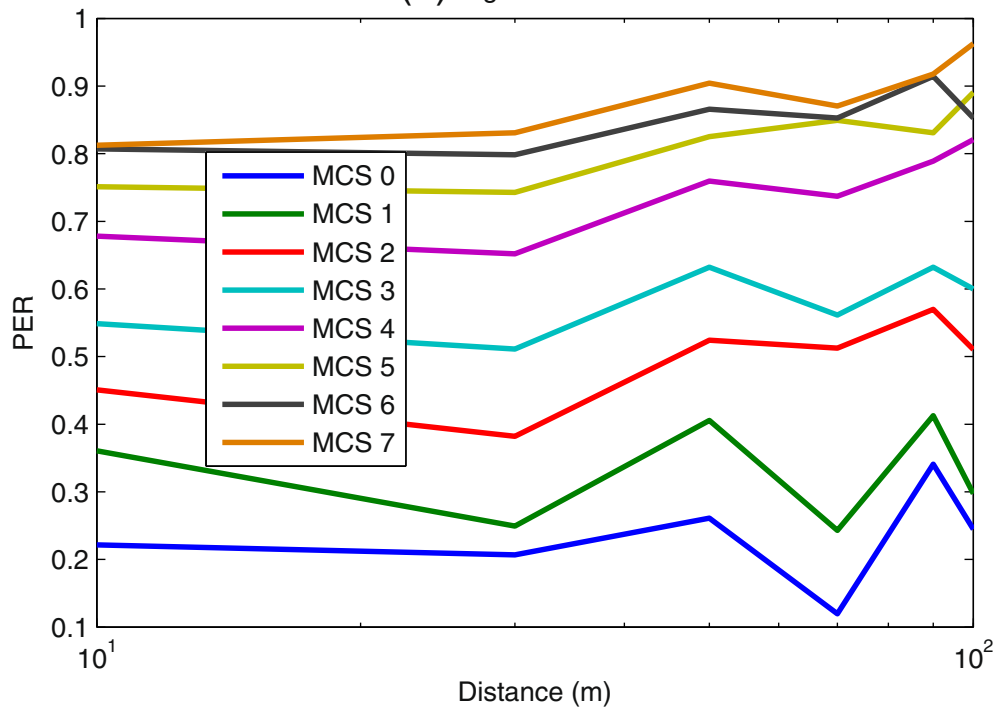

Fig. 14 Average PER versus distance, $k=200, T=1400$ B. a Low multicast data rate. b High multicast data rate 
modes to provide shorter download times at longer distances, i.e. as download time increases with distance, reducing the multicast rate at higher MCS modes results in a lower download time. When we examine the operating range, we find that the multicast data delivery is retained up to a distance of $100 \mathrm{~m}$.

Since we compare different multicast server rates, we also plot the average goodput attained at the application layer before Raptor decoding. Figure 15 shows the results obtained from our hardware trials. It should be noted that the PER and goodput results do not directly reflect the tablet performance (i.e. the average download time) since the Raptor decoding success (and hence the download time) depends on the PER in each source block, rather than the average PER. However, these results provide insights into the understanding of the system limitations. It is seen that a maximum 9-Mbps goodput can be attained using MCS 4 with the lower multicast server rate. Furthermore, MCS 0-3 can achieve higher goodputs if the higher multicast server rate is used (i.e. $18-158 \%$ improvement).

(a) Low multicast rate

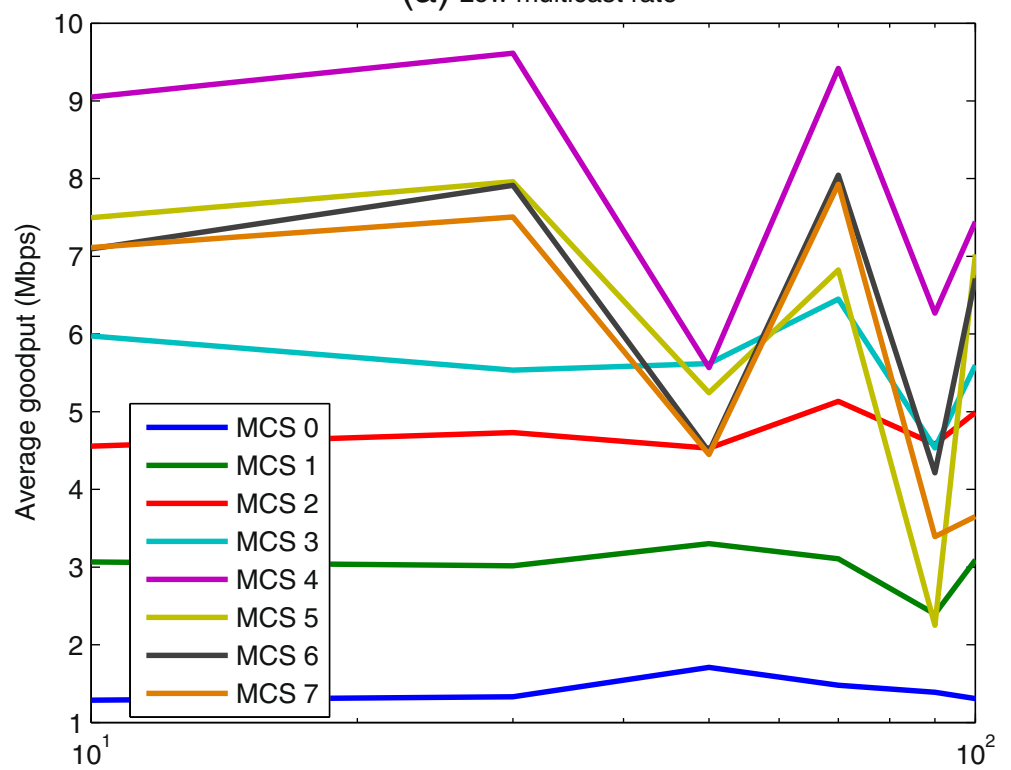

(b) High multicast rate

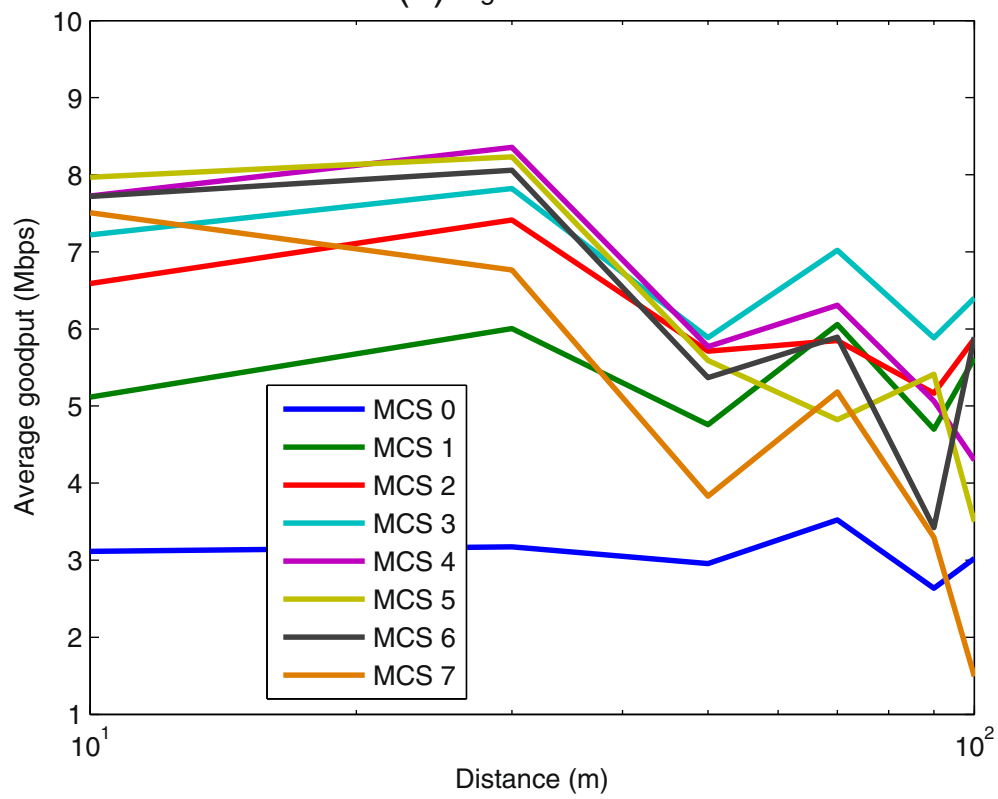

Fig. 15 Average goodput versus distance, $k=200, T=1400$ B. a Low multicast data rate. b High multicast data rate 


\subsection{Analysis of Raptor source block length ( $k$ )}

In this experiment, we evaluate the average download time with respect to different source block sizes $k$ of 50, 200 and 400 for a fixed Raptor source symbol size $T$ of 1400 B. Figure 16 shows the results for MCS modes 1 and 3 . It can be seen that using a small source block size provides shorter download times irrespective of the MCS mode and multicast data rate. However, in theory, for a given file size, increasing the source block size decreases the download time since the probability of retrieving the file correctly increases with $k$ [4]. In our previous work [18], we also evaluated the impact of different source block sizes using the same measured packet traces. The results are consistent with theory; however, neither these results nor the theoretical results take into account the processing limitations of the Wi-Fi client and the effects of the application layer parameters. For example, when we analyse the received packet traces, it is clear that using the larger source block size results in an increase in the UDP PER (Fig. 17) and hence the download time is longer. It is observed that at some locations $k=200$ yields better results than $k=50$; however, our experiments show there is no result when $k=400$ gives a shorter download time than $k=50$ or 200 . This can be attributed to the increased tablet processing effect with increasing $k$. Therefore, based on these observations, it is recommended to use $k \leq 200$ for practical software implementations of RQ of current Android clients.

(a) Low multicast rate

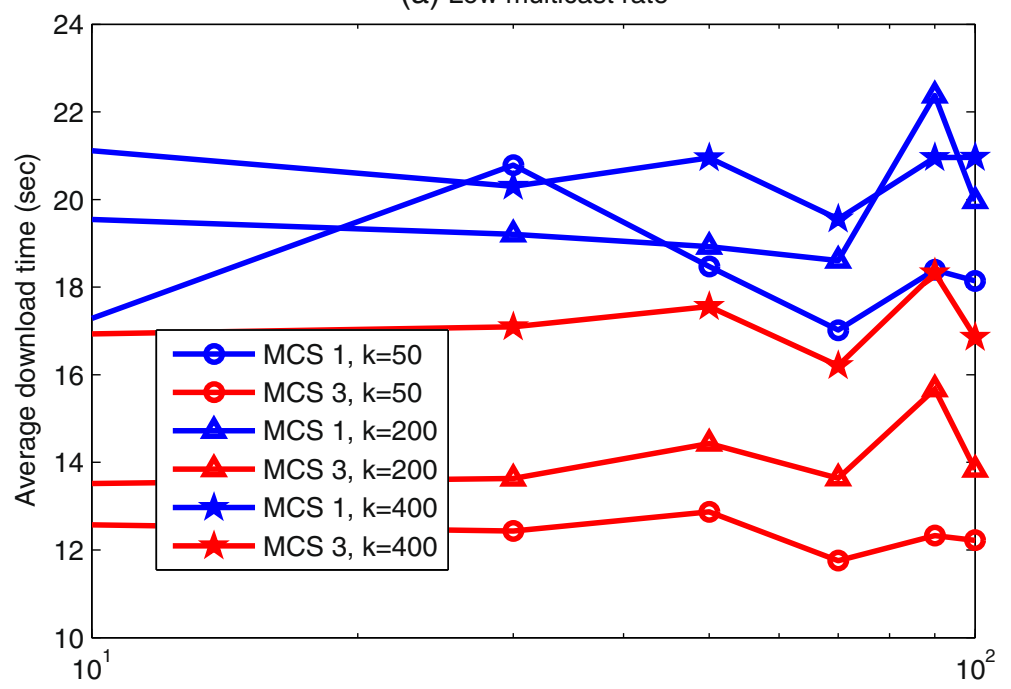

(b) High multicast rate

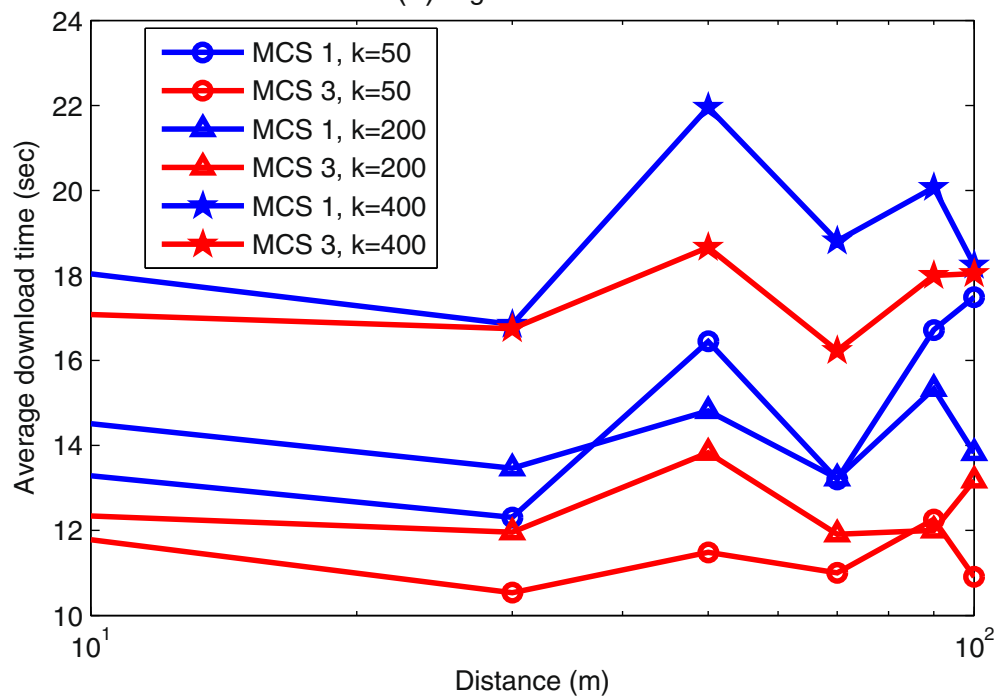

Fig. 16 Average download time versus distance for different source block sizes $k, T=1400$ B. a low multicast data rate, (b) high multicast data rate 
(a) Low multicast rate

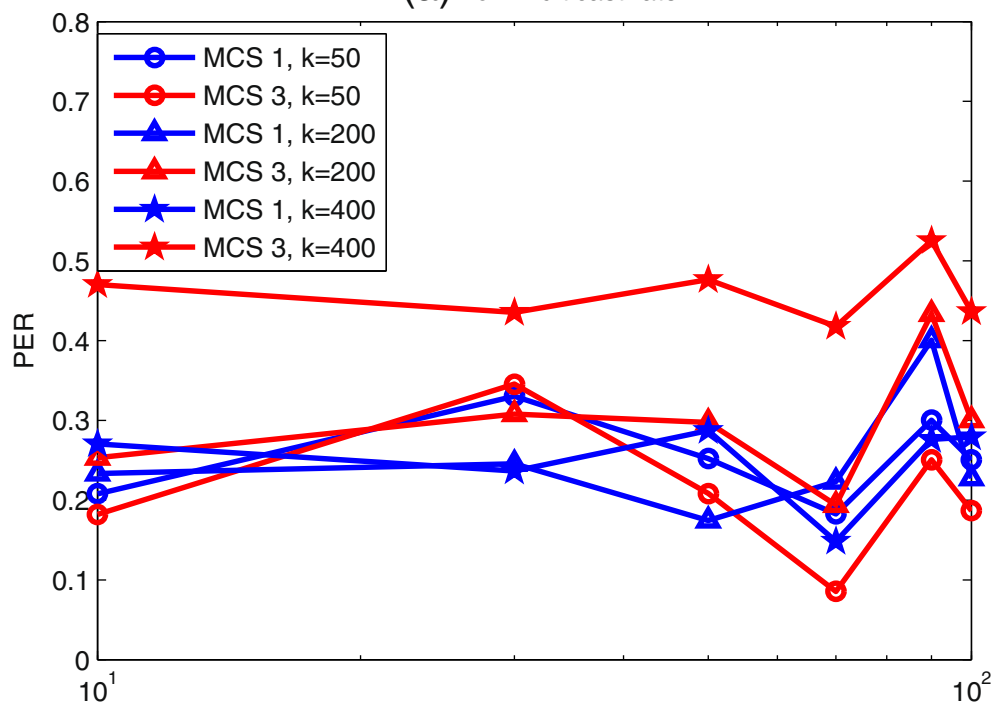

(b) High multicast rate

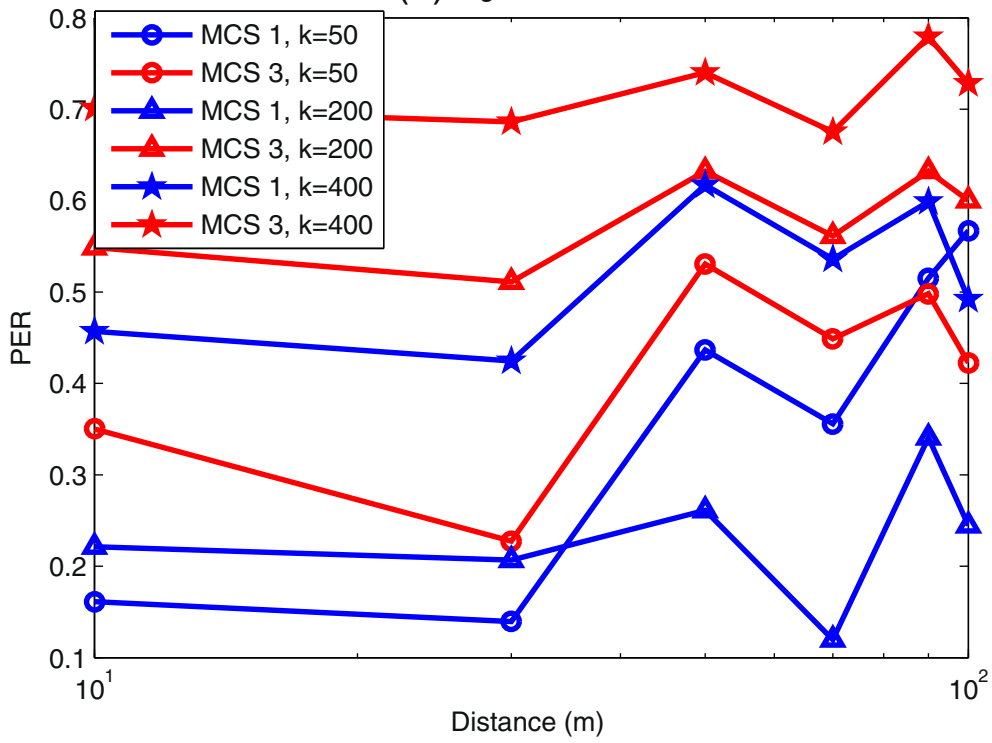

Fig. 17 Average PER versus distance for different source block sizes $k, T=1400$ B. a Low multicast data rate. b High multicast data rate

\subsection{Analysis of Raptor symbols size ( $T$ )}

Another important system design parameter that affects the average download time is the source symbol size $T$. Therefore, in our last experiment, we compare different symbol sizes for a given fixed file and source block size. In this experiment, we consider symbol sizes of 500, 1000 and $1400 \mathrm{~B}$. Measurements were taken for a source block size $k$ of 50 and 200 at the higher and lower multicast server rates. We only present results for $k=200$ and for the higher multicast server rate (the other rates gave similar results). Figures 18 and 19 summarise the results for average download time and PER, respectively. It can be seen that for the same source block size, increasing the symbol size results in shorter download times. This is due to the fact that increasing the symbol size reduces the number of packets to be sent (and this reduces the hardware errors as indicated in Fig. 9a, b) and hence the total number of source blocks. Although small PHY packets are less likely to be corrupted (i.e. the PER is lower) compared to longer packets [27], the errors coming from the tablet dominate the PER at the application layer as seen in Fig. 19. Furthermore, the overheads from the upper layers and PHY also increase as the symbol size decreases. Therefore, it is suggested to use longer packets for devices with limited processing capabilities (e.g. $T \geq 1000 \mathrm{~B}$ ). 


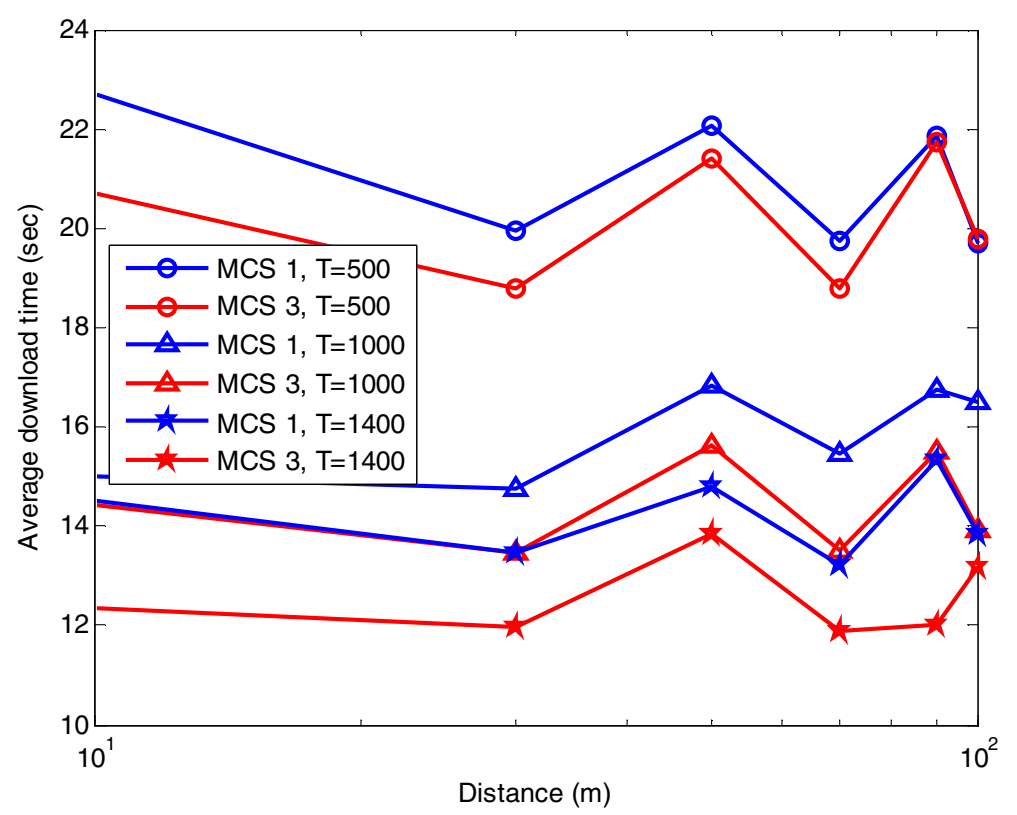

Fig. 18 Average download time versus distance for different source symbol sizes $T, k=200$ and higher multicast data rate

\section{Conclusions}

This paper has presented a reliable and scalable wireless multicast transmission solution for Wi-Fi by combining data carousels with AL-FEC codes. A complete system design, implementation and evaluation process was presented. The results were reported in terms of theoretic simulations and real-world measurements using a server, access point and Android-based client. We developed a detailed multi- layered simulator and compared our simulation results against site-specific measurements from a practical hardware implementation. This paper has presented the first detailed analysis on the implementation of RQ codes and data carousels in a practical Wi-Fi-based server/client system.

Average download time was used as a key performance evaluation metric in order to reflect user QoE. Our results revealed that system performance is mostly

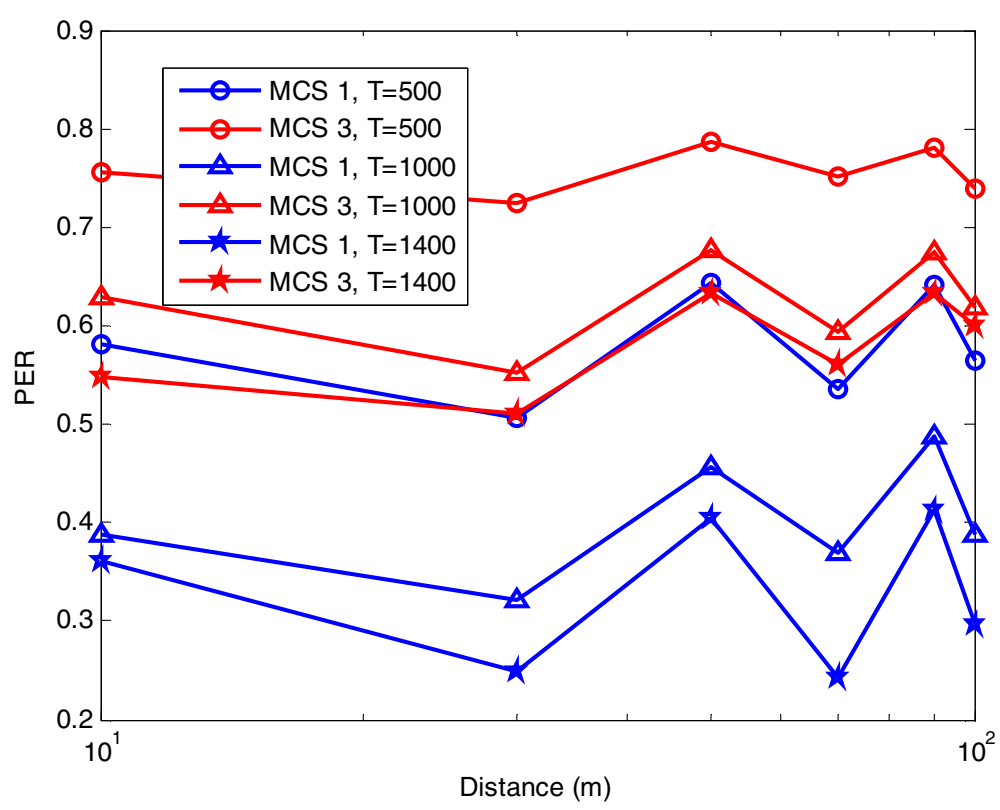

Fig. 19 Average PER versus distance for different source symbol sizes $T, k=200$ and higher multicast data rate 
dominated by tablet limitations. Tablets cannot handle well some of the higher multicast data rates (the maximum goodput in our practical system was around $14 \mathrm{Mbps})$. Moreover, the results showed that for lower and more robust MCS modes (MCS 0-3), using higher multicast server rates provided a shorter download time. However, for higher MCS modes, higher multicast server rates resulted in higher PER at the application layer and, as a result, longer download times. Therefore, for Wi-Fi multicast to tablets, we suggest using lower multicast server rates over higher MCS modes (MCS 4-7).

When we compared Raptor code parameters, we found that unlike theoretic and simulated results, which did not take into consideration packet loss due to hardware limitations, the measurement results showed that small source block sizes gave better results than longer source blocks (i.e. due to the practical limitations of the tablet, using $k \leq$ 200 provided shorter download times). Furthermore, the symbol size also affected the average download time: small symbol sizes resulted in longer download times. This occurred because using a small symbol size increased the number of source symbols and hence the number of source blocks. This increased the processing requirement at the tablet. Therefore, higher symbol sizes (e.g. $T \geq 1000 \mathrm{~B}$ ) are recommended in order to provide higher QoE at the tablet.

This paper has provided a unique insight into the implementation of RQ codes in a practical Wi-Fi multicast data carousel system. We have shown that with suitable design parameters, a scalable high-bandwidth multicast data download service can be offered over Wi-Fi. The hardware and driver limitations of current Wi-Fi clients were shown to play a key role in determining the overall system design. Further investigation into the effects of CPU loading and sleep management is recommended in order to determine areas for improvement.

\section{Competing interests}

The authors declare that they have no competing interests.

\section{Acknowledgements}

The authors would like to thank the Technology Strategy Board (TSB) for part-funding this work as part of the Arkive In the Your Pocket (AIYP) project. The authors are grateful to Broadcom for donating the wireless access points and assisting with the task of multicast parameter optimisation.

\section{Author details}

${ }^{1}$ Department of Electrical and Electronic Engineering, Communications Systems and Networks Group, University of Bristol, Bristol BS8 1UB, UK. ${ }^{2}$ Broadcom UK Ltd, Aztec West, Bristol, UK.

\section{Received: 30 June 2015 Accepted: 27 January 2016}

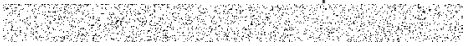

\section{References}

1. IEEE Standard for Information Technology Telecommunications and information exchange between systems Local and metropolitan area networks Specific Requirements Part 11: Wireless LAN MAC and PHY specifications (2012)

2. M Choi, M Samokhina, K Moklyuk, S Choi, J Heo, SJ Oh, VPAL: video packet adaptation layer for reliable video multicast over IEEE 802.11n WLAN. Comput Commun. 33(18), 2271-2281 (2010)
3. S Acharya, R Alonso, M Franklin, S Zdonik, Broadcast disks: data management for asymmetric communication environments. in Proc. ACM SIGMOD Conf. (San Jose,CA, USA, 1995), pp. 199-210

4. M Chipeta, K Karaliopoulos, BG Evans, R Tafazolli, On the use of packet-level FEC and data carousels for the delivery of broadcast/multicast servers to mobile terminals. in Proc. of 61st IEEE Vehicular Technology Conf. (Stockholm, Sweden, 2005), pp. 2394-2399

5. J Peltotalo, S Peltotalo, J Harju, R Walsh, Performance analysis of a file delivery system based on the FLUTE protocol. Int. J. Commun. Syst. 20, 633-659 (2007). doi:10.1002/dac.835

6. I de Fez, F Fraile, R Belda, JC Guerri, Analysis and evaluation of adaptive LDPC AL-FEC codes for content download services. IEEE Trans. Multimedia. 14(3), 641-650 (2012)

7. I de Fez, JC Guerri, An adaptive mechanism for optimal content download in wireless networks. IEEE Trans. Multimedia. 16(4), 1140-1155 (2014)

8. JW Byers, M Luby, M Mitzenmacher, A digital fountain approach to asynchronous reliable multicast. IEEE J. Sel. Areas Commun. 20(8), 1528-1540 (2002)

9. M Luby, T Stockhammer, M Watson, IPTV Systems, Standards and architectures: part II-application layer FEC in IPTV services. IEEE Commun. Mag 46(5), 94-101 (2008)

10. J Heide, MV Perdersen, FHP Fitzek, T Larsen, Network coding for mobile devices-systematic binary random rateless codes. in 4th Int. Mobile Multimedia Commun. Conf. (Oulu, Finland, 2009), pp. 1-6

11. A Shokrollahi, Raptor codes. IEEE Trans. Inf. Theory. 52(6), 2551-2567 (2006)

12. MA Shokrollahi, M Luby, Raptor codes. Foundations Trends Commun. Inf. Theory 6(3-4), 213-322 (2011)

13. 3GPP TS 26346: Multimedia broadcast/multicast server (MBMS): protocols and codecs 2010. http://www.3gpp.org/ftp/specs/archive/26_series/26.346/. Accessed 26.05.2015

14. ETSI TS 102 472: digital video broadcasting (DVB); IP datacast over DVB-H: content delivery protocols 2006. http://www.etsi.org/deliver/etsi_ts/102400_ 102499/102472/0103.01_60/ts_102472v010301 p.pdf. Accessed 26.05.2015

15. Why digital fountain's Raptor code is better than Reed Solomon erasure codes for streaming applications. (Digital Fountain Inc., 2005) https://www. qualcomm.com/media/documents/files/why-raptor-codes-are-better-thanreed-solomon-codes-for-streaming-applications.pdf. Accessed 31May 2015

16. M Luby, L Vicisano, J Gemmell, L Rizzo, M Handley, J Crowcroft, The use of forward error correction (FEC) in reliable multicast. RFC 3452. (Internet Engineering Task Force, 2002), https:/tools.ietf.org/html/ffc3453. Accessed 31.05.2015

17. IEEE 802.11n-2009, amendment 5: enhancements for higher throughput. IEEE Amendment (2009)

18. B Bulut, D Berkovskyy, V Sgardoni, A Doufexi, A Nix, Robust multicast delivery for WLANs: transforming theory into practice, in Int. Conf. on Commun., Man. and Inf. Tech. (ICCMIT). (Prague, Czech Republic, 2015)

19. KH Ng, EK Tameh, A Doufexi, M Hunukumbure, AR Nix, Efficient multielement ray tracing with site-specific comparisons using measured MIMO channel data. IEEE Trans, Veh. Tech 56(3), 1019-1032 (2007)

20. L Wan, S Tsai, M Almgren, A fading-insensitive performance metric for a unified link quality model. IEEE Wireless Commun Netw. Conf. (WCNC) 4, 2110-2114 (2006)

21. M Luby, A Shokrollahi, M Watson, T Stockhammer, RaptorQ forward error correction scheme for object delivery. RFC 6330. (Internet Engineering Task Force, 2011). http://www.ietf.org/ff//fc6330.txt.. Accessed 31.05.2015

22. A Downey, The structural cause of file size distributions. in the 9th Int. Symp. Model. Anal. Simul. Comput. Telecommun. Syst. (MASCOTS). (Cincinnati, OH, USA, 2001), pp. 361-370

23. TS Rappaport, Wireless Communications: Principles and Practice, 2nd edn. (Prentice Hall, New Jersey, 2001)

24. SK Saha, P Deshpande, PP Inamdar, RK Sheshadri, D Koutsonikolas, Powerthroughput tradeoffs of $802.11 \mathrm{n} / \mathrm{ac}$ in smartphones. in Proc. of IEEE INFOCOM. (Hong Kong, 2015)

25. J Jun, P Peddabachagari, M Sichitiu, Theoretical maximum throughput of IEEE 802.11 and its applications. in Proc. of the IEEE Int. Symposium on Network Computing and Applications. (Cambridge, MA, USA, 2003), pp. 249-257

26. R Friedman, A Kogan, Y Krivolapov, On power and throughput tradeoffs of WiFi and Bluetooth in smartphones. IEEE Trans. Mob. Comp. 12(7), 1363-1376 (2013)

27. NF Abdullah, RJ Piechocki, A Doufexi, Systematic Raptor codes for safety broadcast in an unsaturated vehicular highway environment. EURASIP J Wirel. Commun. Netw. 2012, 213 (2012). doi:10.1186/1687-1499-2012-213 